Accepted Manuscript

Mechanical Behavior and Size Effect of the Staggered Bio-Structure Materials

Hansong Ma, Yueguang Wei, Jingru Song, Lihong Liang

PII: S0167-6636(18)30118-2

DOI: 10.1016/j.mechmat.2018.07.009

Reference: MECMAT 2902

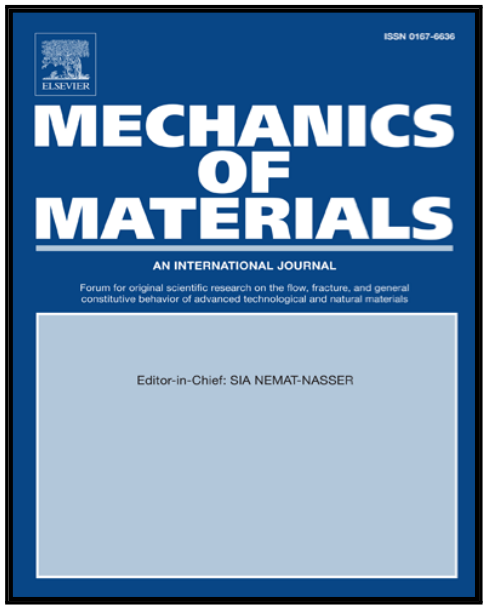

To appear in:

Mechanics of Materials

Received date:

11 February 2018

Revised date:

8 July 2018

Accepted date:

12 July 2018

Please cite this article as: Hansong Ma, Yueguang Wei, Jingru Song, Lihong Liang, Mechanical Behavior and Size Effect of the Staggered Bio-Structure Materials, Mechanics of Materials (2018), doi: 10.1016/j.mechmat.2018.07.009

This is a PDF file of an unedited manuscript that has been accepted for publication. As a service to our customers we are providing this early version of the manuscript. The manuscript will undergo copyediting, typesetting, and review of the resulting proof before it is published in its final form. Please note that during the production process errors may be discovered which could affect the content, and all legal disclaimers that apply to the journal pertain. 


\section{Highlights}

- A strain gradient shear-lag model for the staggered bio-structure is developed in which the effects of microstructures and scale were incorporated.

- The analytical expressions of the overall effective modulus, interfacial strengths and deformations of the staggered bio-structure material are obtained.

- The size effects of the properties of the staggered bio-structure are studied

- The predicted effective moduli of nacreous layer in different shells are compared with corresponding experimental results and are in good agreement with experimental results. 


\title{
Mechanical Behavior and Size Effect of the Staggered
}

\section{Bio-Structure Materials}

\author{
Hansong $\mathrm{Ma}^{\mathrm{a} *}$, Yueguang Wei ${ }^{\mathrm{b} *}$, Jingru Song $^{\mathrm{a}}$, Lihong Liang ${ }^{\mathrm{a}}$ \\ ${ }^{a}$ State-Key Laboratory of Nonlinear Mechanics, Institute of Mechanics, Chinese Academy of Sciences, Beijing \\ 100190, PR China \\ ${ }^{\mathrm{b}}$ College of Engineering, Peking University, Beijing 100871, PR China
}

\begin{abstract}
Staggered structure materials, or "brick and mortar" structure materials, are widely observed in natural biomaterials. In this paper, based on strain gradient theory, a trans-scale shear-lag model is developed to characterize the mechanical properties and their size effects for the staggered bio-structure materials. The analytical expressions of the overall effective modulus, interfacial strengths and deformations of the staggered bio-structure material have been obtained. The results illustrate that the overall effective modulus, interfacial strengths and interfacial deformations have the strong size effects. These effects can be described by a combination parameter, i.e. thickness of organic material layer over the material length scale parameter of strain gradient theory. The overall effective modulus and interfacial strength increase with the decrease of the combination parameter. However, the interfacial deformation considerably decreases with the decrease of the combination parameter value. When the combination parameter value is taken as very large, corresponding to the case of large organic matrix thickness, the results return to the classical ones. The predicted effective moduli of nacre are also compared with experimental results and are in good agreement with experimental results.
\end{abstract}

Keywords: staggered bio-structure; trans-scale model; strain gradient theory; effective modulus; shear-lag model

\footnotetext{
${ }^{*}$ Corresponding authors

E-mail address: mahs@Inm.imech.ac.cn (H. Ma), weivg@pku.edu.cn (Y. Wei)
} 


\section{Introduction}

Natural biomaterials such as nacre, bone, teeth etc. are nanocomposites composed of mineral and organic (Currey, 1977; Jackson et al., 1988; Landis, 1995; Marshall et al., 1997; Tesch et al., 2001; Weiner and Wagner, 1998). Mineral is stiff and brittle, organic is compliant and ductile. Their composites, natural biomaterials, however, exhibit a superior combination of stiffness and toughness compared to their constituents (Barthelat, 2007; Espinosa et al., 2009; Meyers et al., 2008). For example, nacre which is the inner layer of some shells, is composed of $95 \%$ vol. aragonite (a crystallographic form of $\mathrm{CaCO}_{3}$ ) and $5 \%$ vol. organic materials/(proteins and polysaccharides) (Sun and Bhushan, 2012). Its Young's modulus is about $50 \mathrm{GPa}$, comparable to that of mineral (50 100 GPa); its strength is $100 \sim 300 \mathrm{MPa}$, higher than that of mineral $(30 \mathrm{MPa})$; and the fracture toughness is $3 \sim 7 \mathrm{MPa}{ }^{1 / 2}$, which is far higher than that of mineral $\left(\ll 1 \mathrm{MPa} \mathrm{m}^{1 / 2}\right)(\mathrm{Ji}$ and Gao, 2004), and comparable to that of skin (Wegst and Ashby, 2004). Experiments show that fracture energy of nacre is 3000 times more than that of monolithic $\mathrm{CaCO}_{3}$ (Currey, 1977; Jackson et al., 1988). These excellent properties have attracted interests for several decades. And their hierarchical microstructures have been identified as the key factor for the outstanding properties (Currey, 1977; Dunlop and Fratzl, 2010; Ji and Gao, 2004).

The microstructure in biomaterials is hierarchy and spans several length scales (Dunlop and Fratzl, 2010). For example, there are 3 levels structure in shells from nanoscale to macroscale (Currey, 1977; Jackson et al., 1988; Menig et al., 2000). A shell generally consists of periostracum layer (composed of hardened protein), prismatic layer (composed of columnar calcite) and nacreous layer (Sun and Bhushan, 2012), where nacre holds the majority of volume. The typical microstructure of nacre is a "brick and mortar" structure, in which bricks or mineral platelets are arranged in a staggered micro-array and "glued" together with organic materials (Sun and Bhushan, 2012). The thickness of mineral platelets is $0.2 \sim 1 \mu \mathrm{m}$ and that of "glued" organic layers is only 20 40 nm (Barthelat et al., 2007; Jackson et al., 1986; Wang et al., 2001). The mineral platelets are also composites composed of aragonite nanocrystals 
about 3-10 nm embedded in a continuous organic material (Zhang et al., 2016). Bone has more than 7 levels structure from nanoscale to macroscale (Dunlop and Fratzl, 2010; Weiner and Wagner, 1998). Although these complicated structural geometries, the nanoscale elementary structure and the staggered bio-structure are involved in their basic building blocks ( $\mathrm{Ji}$ and Gao, 2010), such as the mineralized collagen fibrils in bone (Weiner and Wagner, 1998) and teeth (Kinney et al., 2003). The study of the relation between these microstructures and the unique overall mechanical properties is helpful for the successful design of biomimetic materials.

Mechanical models for the relation between the microstructure and the overall properties have been studied for many years. According to the observed microstructure of nacre, Currey (1977) proposed a "brick and mortar" model to connect the microstructure and the overall mechanical properties of nacre. Based on the observed mineral bridges in red abalone (Song et al., 2003), which immersed in the organic interlaminations and connected two individual mineral platelets, Song et al. (2003) introduced them into the mechanical model. Similarly, the observed nanoasperities on the mineral platelets (Wang et al., 2001), the mineral platelet waviness (Barthelat et al., 2007), even the combinations of these microstructure features were also involved in mechanical models (Sun and Bhushan, 2012). All of the above mechanical models were established by including the actual observations of microstructure.

Jäger \& Fratzl (2000) developed a mechanical model with a staggered array of mineral platelets for collagen fibrils by considering tensile and shear deformations of the organic matrix. The results showed that when the thickness of organic layers decreased, the mechanical behavior started to be dominated by shear of organic layers. Dai et al. (2008) modified this model by including the deformation of mineral platelets. Based on the work of Jäger \& Fratzl (2000), Gao et al.(2003) and Ji \& Gao (2004) proposed a tension-shear-chain (TSC) model by considering tensile deformation in mineral platelets and uniform shear in organic matrix and they derived a compact formula of effective modulus. They pointed that the large aspect ratio and staggered structure were the important factors for the large stiffness of biomaterials. 
Zhang et al. (2010) extended this model to randomly staggered platelets distribution. Zuo \& Wei (2007) modified TSC model by considering non-uniform shear in organic matrix, which was called shear-lag model (SL). It was used to study the mechanical properties of bone-like hierarchical materials (Gao, 2006) and the results showed that when the number of hierarchy levels was large, the non-uniform of shear in organic matrix must be considered. Based on the shear-lag assumptions also, Bar-On and Wagner (2011) proposed a generic staggered structure mechanical model by considering the tensile and shear deformations of mineral platelets and organic matrix. Although all of the above models catch the main characteristics of staggered structure, they contain neither the microstructure features (mineral bridges, nanoasperities, etc.) nor the nanoscale characteristics. Their predicted results are suitable for a mineral platelet of aspect ratio larger than 30 ( $\mathrm{Ji}$ and Gao, 2004) or depend on the accurate experimental measurements of the properties of organic matrix. For example, the used modulus of organic matrix in nacre in literature (Bar-On and Daniel Wagner, 2012) is $3 \mathrm{GPa}$ (Barthelat et al., 2007). When the experimental measured value is small, such as $20 \mathrm{MPa}$ in literature (Katti et al., 2001) on nacre, the resulted effective modulus is $20 \mathrm{GPa}$ (Katti et al., 2001), which is lower than actual value.

Therefore, in this paper, a trans-scale model will be developed to contain the effects of microstructure and nanoscale for a staggered bio-structure based on the following considerations:

1). The staggered bio-structure spans several length scale from nanoscale to macroscale. The scale features play an important role so that they cannot be neglected. Thereby its overall properties should be characterized by the trans-scale mechanical theory to demonstrate the effects of scale.

2). Because the organic layers in staggered bio-structure are very thin and there are mineral bridges, nanoasperities and waviness of platelets immersed in them, the organic layers are difficult to deform (Barthelat et al., 2007) so that the measured modulus of organic materials in interlaminations (e.g., 3 GPa (Barthelat et al., 2007)) are much higher than that of general organic materials, which is $50 \sim 100 \mathrm{MPa}$ (Ji and Gao, 2004). When the thickness of organic layers increases, the effects of these 
immersed microstructure features are decreased so that the organic layers will gradually soften to bulk cases. That is, if we regard the organic material and the immersed microstructure features as an effective medium, its modulus should be size-dependence. Therefore, the organic matrix can be characterized by scale theory, i.e., high order continuum theory, which incorporated the effects of microstructure and scale.

3). The thinner the organic layers, the rapider the strain change in it, the stronger the strain gradient effect is.

So, in this paper, the organic materials will be regarded as strain gradient continuum, then the Zuo \& Wei's shear-lag model (Zuo and Wei, 2007), which is called classical shear-lag model in the following, will be extended to characterize the effective modulus of staggered structure in biomaterials. The paper is arranged as follows, in Section 2, the trans-scale model is established. In Section 3, the theoretical model will be solved, and the results are presented to illustrate the characteristics of the proposed model and some conclusions are summarized finally. In the following, subscript "m" means the organic matrix, and "p" means the mineral platelet.

\section{The Model}

The considered staggered bio-microstructure is shown in Fig.1. Inside an organic material matrix, mineral platelets with length $L$ and thickness $h$ are arranged periodically, the neighboring mineral platelets are staggered by overlapping a half of their length. The thickness between the two layers of mineral platelet, i.e. the organic layers, is $d$. The mineral platelets are considered still as elastic material which will undergo the very small deformation due to their very high stiffness compared with organic material. However the organic layers are regarded as strain gradient continuum because of their very small size in thickness and thickness size-dependence of mechanics properties. The fundamental assumptions for the establishments of tension-shear-chain model (Gao et al., 2003; Ji and Gao, 2004) and the classical shear-lag model (Zuo and Wei, 2007) are followed:

1). The modulus of mineral platelets is about 3 orders higher than that of organic 
matrix so that the tensile stress is carried mainly by platelets and the shear stress is carried mainly by organic materials. Under a tensile load, the path of load transfer is shown in Fig.1 (b).

2). The length of mineral platelet is one or two orders larger than its thickness so that the deformation is one dimensional, that is, depending only on $x$, Fig.1 (c), and the stress in mineral platelets can be regarded as unchanged across its thickness.

3). The gap between the ends of two mineral platelets is much smaller than the length of mineral platelets such that the mechanical load at this zone is ignored. Therefore, the volume fraction of mineral platelets $\phi$ is defined by $\phi=h /(d+h)$.

4). The elastic strain gradient organic matrix layers are very long in $x$ direction and very thin in $y$ direction so that an additional condition is assumed: the shear stress in the organic matrix is non-uniform, and only the strain gradient effect in $y$ direction is considered, and the strain gradient effect in $x$ direction is neglected.

It is worth noting that for the present case of nacre, since the Young modulus of the mineral platelets is much bigger than that of the proteins, the modulus ratio of both layers is about 1000 times, and the deformation of the mineral platelets is much smaller than that of the protein layers, therefore, the strain gradient effect in mineral platelets can be neglected. Moreover, since the ratio of matrix layer thickness to its length $(d / L)$ is very small, about 0.01 , according to quantity level analysis, the derivative of any variable to coordinate $\mathrm{x}$ is much smaller than its derivative to coordinate $y$, so we can neglect the strain gradient effect in the $\mathrm{x}$ direction to be compared with/its effect in the y direction.

Considering the symmetry, a representative cell is taken into account, as shown in Fig.1 (c), which is a quarter of a repeat cell. Due to the effect of elastic strain gradient, the deflection angle of the organic matrix is no longer uniform with the value of $y$ under the tensile load in Fig.1 (c). 


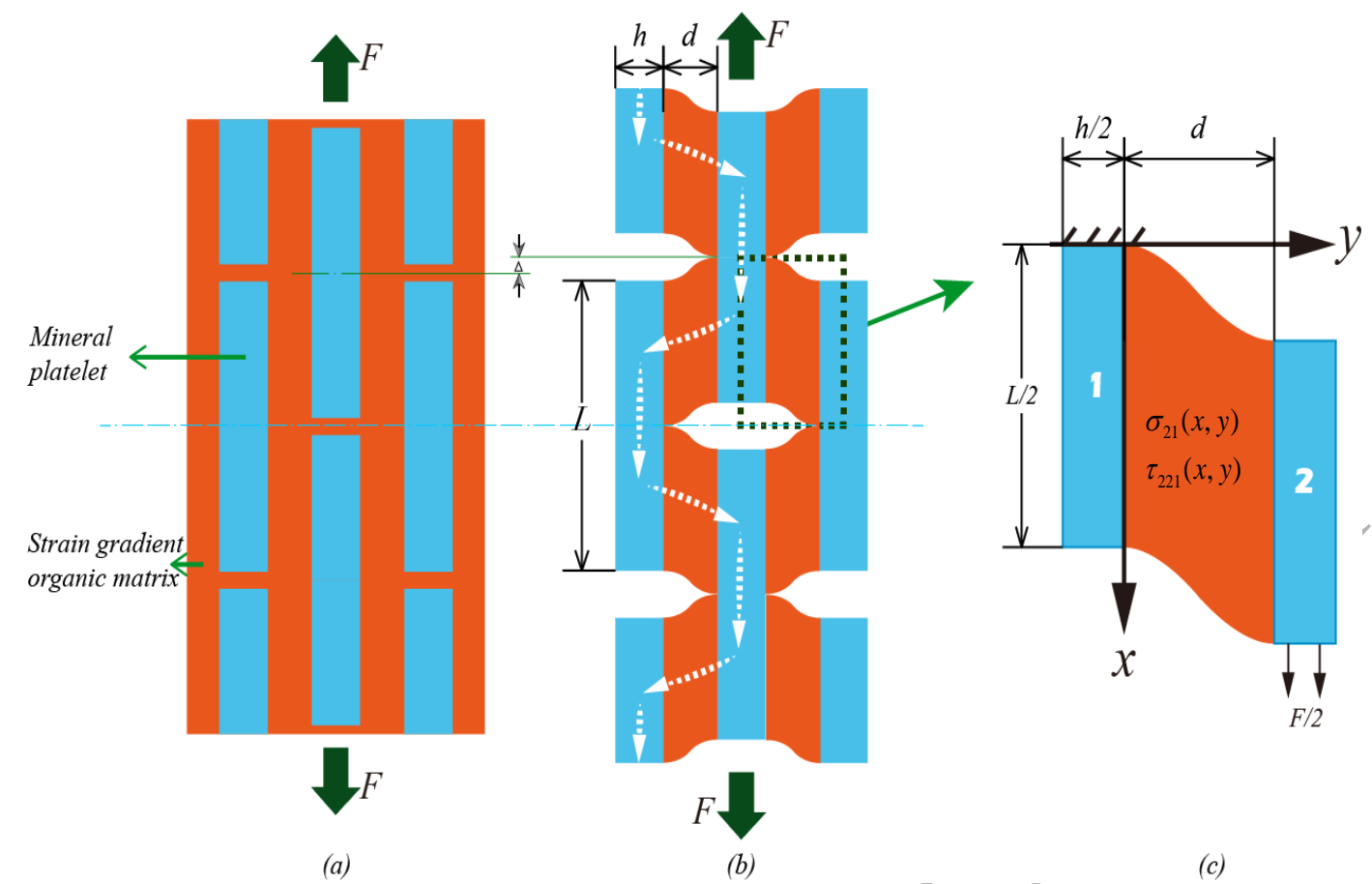

Fig.1 the schematic diagram of strain gradient shear-lag model

In characterization of the elastic strain gradient effect, the simplified form of Wei-Hutchinson strain gradient theory (Song et al., 2014; Wei, 2006; Wei and Hutchinson, 1997) is used to describe the organic matrix materials. Its basic equations are written as:

$$
\begin{aligned}
& \varepsilon_{i j}=\frac{1}{2}\left(u_{i, j}+u_{j, i}\right) \kappa_{i j \bar{k}}=\nabla \nabla \mathbf{u}=u_{, k} \bar{i} \kappa \\
& \sigma_{i k, i}-\tau_{i j k, i j}=0 \\
& \sigma_{i j}=\lambda \varepsilon_{k k} \delta_{i j}+2 \mu \varepsilon_{i j} \quad \tau_{i j \bar{k}}=2 E l^{2} \kappa
\end{aligned}
$$

where $u_{i}$ is the displacement vector, $\varepsilon_{i j}$ and $\kappa_{i j k}$ are the strain and strain gradient tensors, $\sigma_{i j}$ and $\tau_{i j k}$ are the stress and high-order stress tensors, which are the work conjugate to the $\varepsilon_{i j}$ and $\kappa_{i j k}$ respectively. $\lambda, \mu$ are the Lame coefficients, $E$ is the Young's modulus, $\delta_{i j}$ is Kronecker delta, $l$ is the material length scale parameter.

The principle of virtual work is:

$$
\int_{V}\left(\sigma_{i j} \delta \varepsilon_{i j}+\tau_{i j k} \delta \kappa_{i j k}\right) d V=\int_{V} f_{i} \delta u_{i} d V+\int_{S} t_{i} \delta u_{i} d S+\int_{S} r_{i}\left(D \delta u_{i}\right) d S
$$


where $d V$ and $d S$ are the volume and surface area elements, $f_{i}$ is the body force per unit volume, $t_{i}$ is the surface traction and $r_{i}$ is the double stress traction on the surface $S$. On the surface $S$, the gradient $u_{k, j}$ can be decomposed into a surface-gradient $D_{j} u_{k}$ and a normal gradient $n_{j} D u_{k}$, that is, $u_{k, j}=D_{j} u_{k}+n_{j} D u_{k}$, where $D_{j}=\left(\delta_{j k}-n_{j} n_{k}\right) \partial_{k}$ and $D=n_{i} \partial_{i}, n_{i}$ is the unit normal cosines on the surface $S$. The surface traction $t_{i}$ and the double-stress traction $r_{k}$ are:

$$
t_{k}=n_{\imath}\left(\sigma_{i \bar{k}} \partial \tau_{j}\right)_{i j} \underset{k}{\dagger} n n \tau_{j}\left({ }_{i} D_{l j k} n\right)-_{p} D_{p}(\tau), \quad r_{k}=n_{i} n_{j} \tau_{i j k}
$$

Then for the staggered structure and simplified model shown in/Fig.1, the stress and high-order stress of the strain gradient organic matrix are:

$$
\sigma_{21}=2 G_{m} \varepsilon_{21}=G_{m} \frac{\partial u_{m}}{\partial y}, \quad \tau_{221}=2 E_{m} l^{2} \kappa_{221}=2 E_{m} l^{2} \frac{\partial^{2} u_{m}}{\partial y^{2}}
$$

where $u_{m}$ is the displacement of the organic matrix in $x$ direction. $G_{m}$ and $E_{m}$ are the shear and Young's moduli of the organic matrix.

The mineral platelets in the staggered bio-structure are considered as traditional linear elastic materials. So they satisfy the following equations:

$$
\sigma_{i}=E_{p} \varepsilon_{i}=E_{p} \frac{d u_{p i}}{d x}
$$

where $u_{p i}$ is the displacement of the platelet $i(i=1,2)$ in Fig.1, $E_{p}$ is the Young's modulus of mineral platelets.
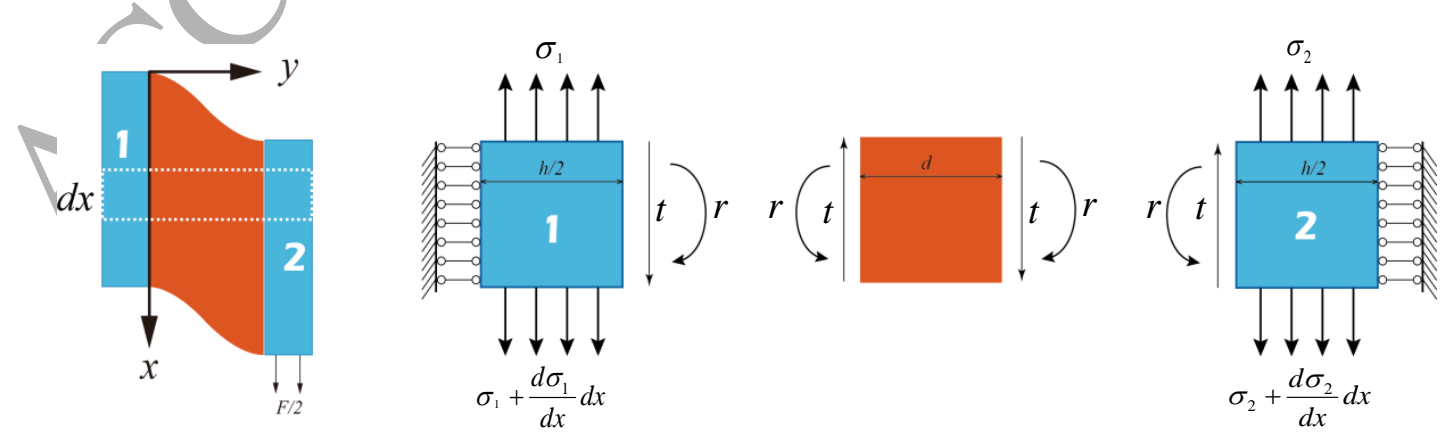

Fig.2. Forces analysis for cell

The forces analysis of the representative volume cell is shown in Fig.2. $t, r$ are 
the surface traction and double-stress traction at the $y=0, d$ interfaces between the mineral platelets and the organic matrix, respectively. For convenience, the subscript " 1 " in $t_{1}, r_{1}$ has been omitted here since only the component in 1 direction is not 0 .

For the platelet 1 and 2, the moment is balanced by the surface traction and double-stress traction on the other half platelet. And the force equilibrium conditions give the following two balance equations:

$$
\left\{\begin{array}{l}
\frac{E_{p} h}{2} \frac{d^{2} u_{p 1}(x)}{d x^{2}}+t=0 \\
\frac{E_{p} h}{2} \frac{d^{2} u_{p 2}(x)}{d x^{2}}-t=0
\end{array}\right.
$$

where

$$
t=\sigma_{21}-\partial_{2} \tau_{221}=G_{m} \frac{\partial u_{m}}{\partial y}-2 E_{m} l^{2} \frac{\partial^{3} u_{m}}{\partial y^{3}}
$$

is obtained by Eqs.(2.5), (2.6) and the gradient in $x$ direction is neglected.

The boundary conditions are:

$$
\begin{aligned}
& \text { at the boundary } x=0:\left.\quad u_{p 1}\right|_{x=0}=0,\left.\quad \sigma_{2}\right|_{x=0}=0, \\
& \text { at the boundary } x=\frac{L}{2}:\left.\quad \sigma_{1}\right|_{x=L / 2}=0,\left.\quad \sigma_{2}\right|_{x=L / 2}=\frac{F}{h}=\sigma_{\max },
\end{aligned}
$$

To solve this problem, a compatibility relation between $u_{p 1}(x), u_{p 2}(x), u_{m}(x, y)$ is needed and it ean be obtained from the interfaces continuous conditions.

We assume that no work is done at the interfaces $y=0, d$ so that the displacement $u_{i}$ and the normal gradient $D u_{i}$ are continuous at the interfaces according to the virtual work principle (2.4) (Fleck and Hutchinson, 1993). That is:

$$
\begin{aligned}
& \text { at interface } y=0:\left.\quad u_{m}\right|_{y=0}=u_{p 1}, \quad D u_{m}=D u_{p 1} \Rightarrow \frac{\partial u_{m}}{\partial y}=\frac{\partial u_{p 1}}{\partial y}=0 \\
& \text { at interface } y=d:\left.\quad u_{m}\right|_{y=d}=u_{p 2}, D u_{m}=D u_{p 2} \Rightarrow \frac{\partial u_{m}}{\partial y}=\frac{\partial u_{p 2}}{\partial y}=0
\end{aligned}
$$

Using Eqs. (2.9), (2.11), we obtain: 


$$
\begin{gathered}
u_{m}=u_{p 1}+\frac{\left(1-e^{A d}\right)}{2\left(1-e^{A d}\right)+A d\left(1+e^{A d}\right)}\left(u_{p 2}-u_{p 1}\right)+\frac{A\left(1+e^{A d}\right)}{2\left(1-e^{A d}\right)+A d\left(1+e^{A d}\right)}\left(u_{p 2}-u_{p 1}\right) y \\
-\frac{1}{2\left(1-e^{A d}\right)+A d\left(1+e^{A d}\right)}\left(u_{p 2}-u_{p 1}\right) e^{A y}+\frac{e^{A d}}{2\left(1-e^{A d}\right)+A d\left(1+e^{A d}\right)}\left(u_{p 2}-u_{p 1}\right) e^{-A y}
\end{gathered}
$$

and

$$
t=\frac{G_{m}}{d\left[1-\frac{2\left(e^{A d}-1\right)}{A d\left(e^{A d}+1\right)}\right]}\left(u_{p 2}-u_{p 1}\right)
$$

where $A=\sqrt{\frac{G_{m}}{2 E_{m} l^{2}}}=\frac{1}{2 l \sqrt{1+v_{m}}}=\frac{\beta}{2 d \sqrt{1+v_{m}}}, v_{m}$ is the Poisson ratio of the organic matrix , and $\beta=d / l$ is a dimensionless length scale parameter for the effect of strain gradient. When $\beta=d / l \rightarrow 0$, then $\frac{2\left(e^{A d}-1\right)}{A d\left(e^{A d}+1\right)} \rightarrow 1$, Eqn.(2.13) can be rewritten as $\left(u_{p 2}-u_{p 1}\right)=0$. It means that the strain gradient effect is so strong that it makes the strain gradient organic matrix too stiff to deform, which is the same effects as the immerged mineral bridges, nanoasperities. When $\beta=d / l \rightarrow \infty$, then $\frac{2\left(e^{A d}-1\right)}{A d\left(e^{A d}+1\right)} \rightarrow 0$, Eqn. $(2.13)$ is reduced to the traditional relation of the shear-lag model for the staggered bio-structure (Zuo and Wei, 2007). It means when the thickness of the strain gradient organic matrix $d$ is far greater than the strain gradient characteristic length $l$, the strain gradient effect is so small that it can be neglected. It is exactly the feature of scale.

By Eqs.(2.8), (2.9), (2.10), (2.12), we can obtain the displacements of the mineral platelets and the surface traction:

$$
\begin{aligned}
& u_{p 1}(x)=\frac{\sigma_{\max } L}{2 \eta E_{p}}\left[\frac{e^{\eta / 2}+1}{e^{\eta / 2}-1}+\frac{x \eta}{L}-\frac{1}{e^{\eta / 2}-1} e^{\eta x / L}-\frac{e^{\eta / 2}}{e^{\eta / 2}-1} e^{-\eta x / L}\right] \\
& u_{p 2}(x)=\frac{\sigma_{\max } L}{2 \eta E_{p}}\left[\frac{e^{\eta / 2}+1}{e^{\eta / 2}-1}+\frac{x \eta}{L}+\frac{1}{e^{\eta / 2}-1} e^{\eta x / L}+\frac{e^{\eta / 2}}{e^{\eta / 2}-1} e^{-\eta x / L}\right]
\end{aligned}
$$




$$
t(x)=\frac{\eta \sigma_{\max }}{4 \rho}\left[\frac{1}{e^{\eta / 2}-1} e^{\eta x / L}+\frac{e^{\eta / 2}}{e^{\eta / 2}-1} e^{-\eta x / L}\right]
$$

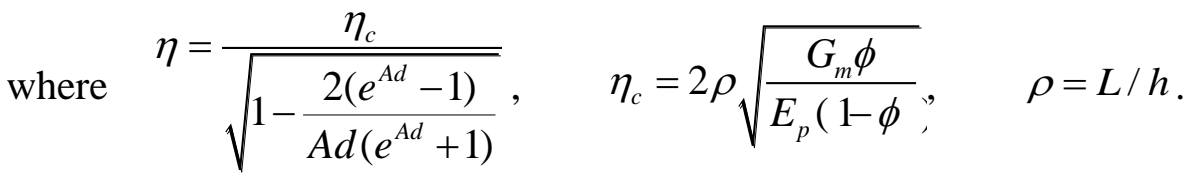

The stress fields of the mineral platelets and the strain gradient organic matrix can be obtained by Eqs. (2.6), (2.7), which are listed in Appendix A.

The effective Young's modulus $E_{c}$ of the staggered bio-structure is defined as the ratio of effective stress to effective strain of the representative cell. Then the analytical expression of the effective modulus of the staggered bio-structure is:

$$
\frac{1}{E_{c}}=\frac{\bar{\varepsilon}}{\bar{\sigma}}=\frac{\frac{\Delta}{L / 2}}{\frac{F}{2(h+d)}}=\frac{1}{\phi E_{p}}+\frac{4}{\eta \phi E_{p} \tanh \left(\frac{\eta}{4}\right)}
$$

where $\bar{\varepsilon}, \bar{\sigma}$ are the effective strain and stress, $F=h \sigma_{\max }, \Delta$ is the elongation of the half representative cell and $\Delta=u_{p 2}\left(\frac{L}{2}\right)-u_{p 1}(0)$.

When $\beta=d / l \rightarrow \infty, \eta \rightarrow \eta_{c}$, the Eqn.(2.17) is reduced to the expression of effective Young's modulus derived by the classical shear-lag model (Zuo and Wei, 2007).

\section{Results and analysis}

In this section, we shall discuss the mechanical behavior and size effect for the staggered bio-structure materials based on the above fundamental displacement and stress solutions given by Eqns.(2.6) (2.17). Since the strain gradient theory can be used to describe the mechanical behavior of materials at a hundred nanometer scale through length scale $l$ (with magnitude about one micron), so by means of the present model we have analyzed the mechanical behavior of the bio structure at the nanoscale as $\beta=d / l$ value is approaching about 0.04 (for the nacre case: volume fraction $95 \%$, $L \sim 5$ micron, $h \sim 1$ micron, $d \sim 0.04 l \sim 40$ nanometer). 


\subsection{Neglecting the mineral platelet deformation}

Since the modulus ratio of organic matrix to mineral platelet is very small, we first consider a simple case when the deformations of mineral platelets relative to those of organic matrix can be neglected. In this case, one can directly take as $u_{p 1}=0$ and obtain a constant surface traction, $t=F / L$. The displacement and stress solutions can be degenerated from fundamental solutions by setting $E_{p} \rightarrow \infty$. Then the displacement of organic matrix can be given as:

$$
u_{m}=u_{p 2 c} \frac{1-e^{A d}+A\left(1+e^{A d}\right) y-e^{A y}+e^{A d} e^{-A y}}{A d\left(1+e^{A d}\right)}
$$

where $u_{p 2 c}=\frac{F d}{L G_{m}}$ is the conventional displacement of platelet 2, which can be obtained by the classical shear-lag model with rigid mineral platelet case.

Fig. 3 shows the variation of the normalized displacement of the organic matrix $u_{m} / u_{p 2 c}$ along axis $y$ with different $\beta=d / l$ in the case of rigid mineral platelets. It can be seen that the smaller the $\beta$ value, the smaller the $u_{m} / u_{p 2 c}$ value. It illustrates the size effect of organic layer deformation. On the other hand the solution shows that considering the strain gradient effect makes the organic matrix stiff and difficult to deform. When $\beta=d / l$ is large enough, the result tends to the classical one, i.e. $u_{m c} / u_{p 2 c}=y / d$. Moreover, considering strain gradient effect, $u_{m} / u_{p 2 c}$ is no longer linear along $y$ axis. 


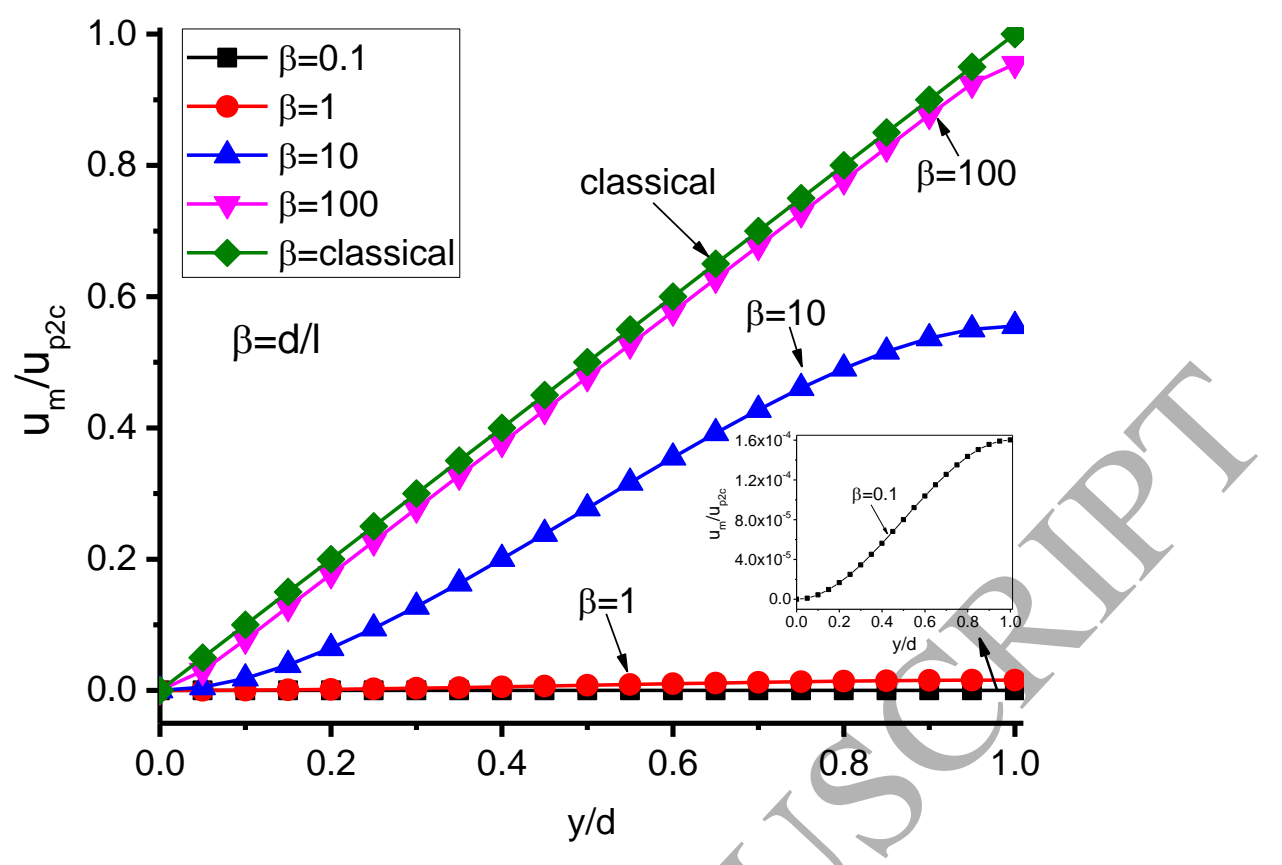

Fig.3. Variation of $u_{m} / u_{p 2 c}$ along $y$ axis with different $\beta$ in the case of rigid mineral platelets

\subsection{Considering the mineral platelet deformation}

In order to investigate the overall properties of the staggered structure, consider that the mineral platelet is elastic material and its deformation is not neglected. The deformation mechanism of the staggered structure model is also referred to Fig.1.

The variations of the traction $t$ given in Eqn.(2.16) are examined firstly. In the following calculations, the Poisson's ratio of the organic matrix is set as $v_{m}=0.3$ since it has little influence on the solutions due to quasi one-dimension simplification of the proposed model.

The variations of traction $t$ as a function of $x, \rho, \phi$ with different $\beta=d / l$ are illustrated in Figs.4 (a), (b), (c) and (d) respectively. From Fig.4 (a), the traction $t$ increases at the two ends of the elastic mineral platelets with the decrease of $d$ ( $l$ is material length scale, material constant, its value is about the order of 1 micron). The smaller the $d$ value, the larger the traction value. On the other hand, when $d$ is large enough, the result returns to the classical one. From Fig.4 (b), the stain gradient effect has large influences on the traction $t$ when the aspect ratio $\rho$ is small and at 
$x=0.04 L$. It is to say that the traction $t$ has the big size effect when the aspect ratio $\rho$ is small. And the value of $t$ decreases and tends to 0 with the increase of $\rho$ at $x=0.04 L$ when $\phi=0.95, E_{m} / E_{p}=0.001$. Fig.4 (c) demonstrates the effect of the volume fraction $\phi$ on the surface traction $t$ with $\rho=5, x=0.04 L, E_{m} / E_{p}=0.001$. It is found that in the classical shear-lag model, the volume fraction $\phi$ has small effect on the traction $t$, however when the strain gradient effect is involved, the volume fraction $\phi$ has a very large effect on the traction $t$. Fig.4 (d) displays the overall stress-strain relations for $\rho=5, x=0.04 L, E_{m} / E_{p}=0.001$ and for several $\beta=d / l$ values from 0.04 to 10 . From Fig.4(d), both the effective strength and stiffness (slopes of stress-strain relations ) have the obvious size effects of the matrix thickness $d$ for changing $d / l$ values within region of $0.04 \sim 10$.

The strain gradient effects cause a large influence on the traction $t$ and consequently on the mineral platelet stress and deformation. These effects are displayed in Figs.5 (a) and (b). From Figure 5, we can also observe the big size effects of the displacement and stress existing in the mineral platelet 1 .

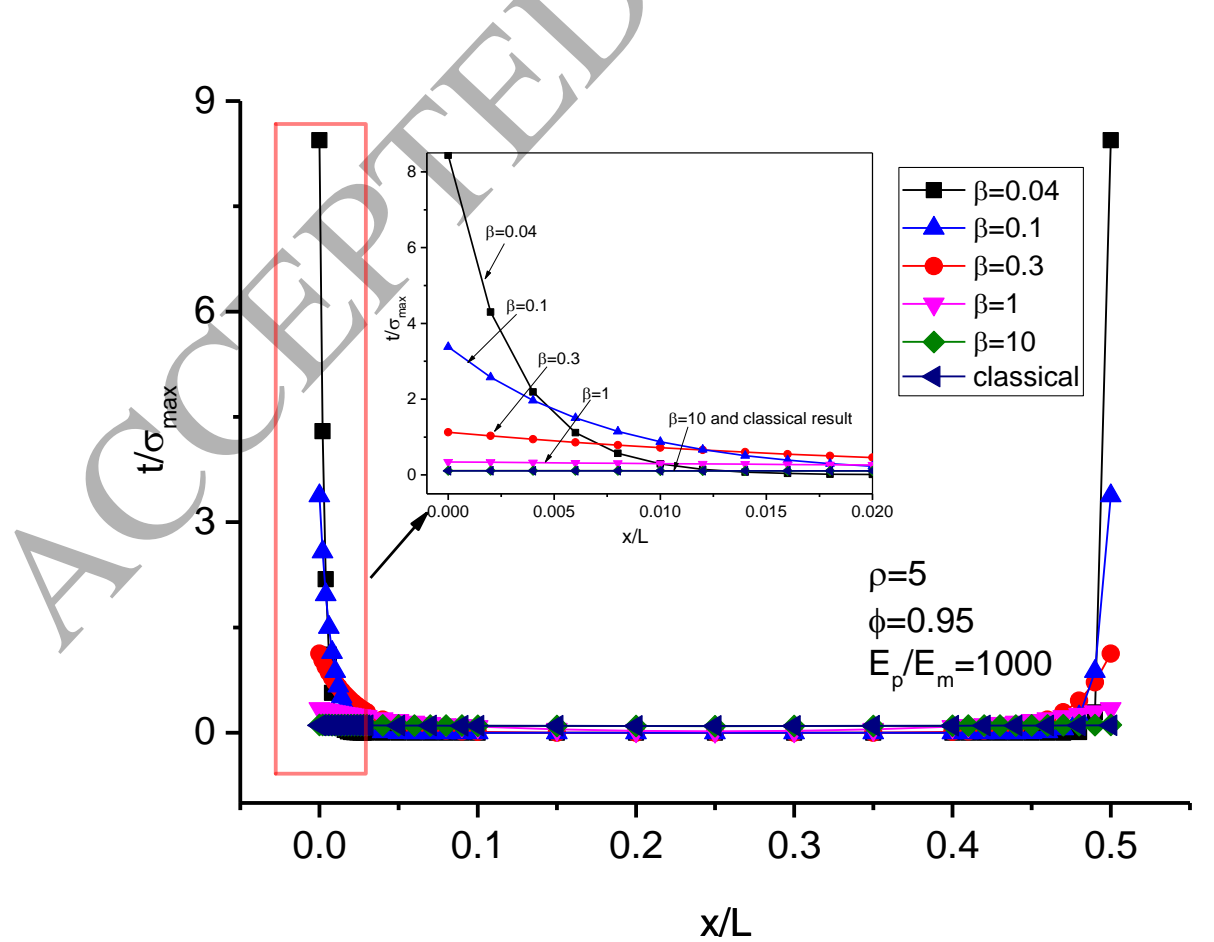


(a)

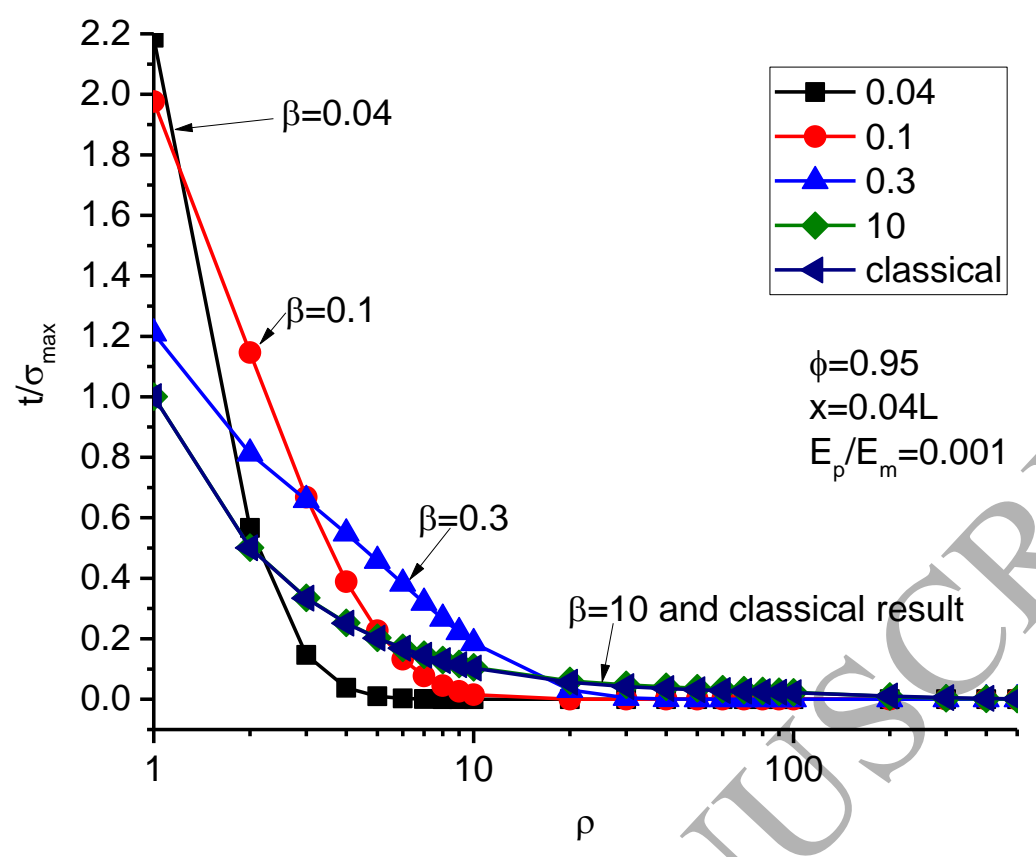

(b)

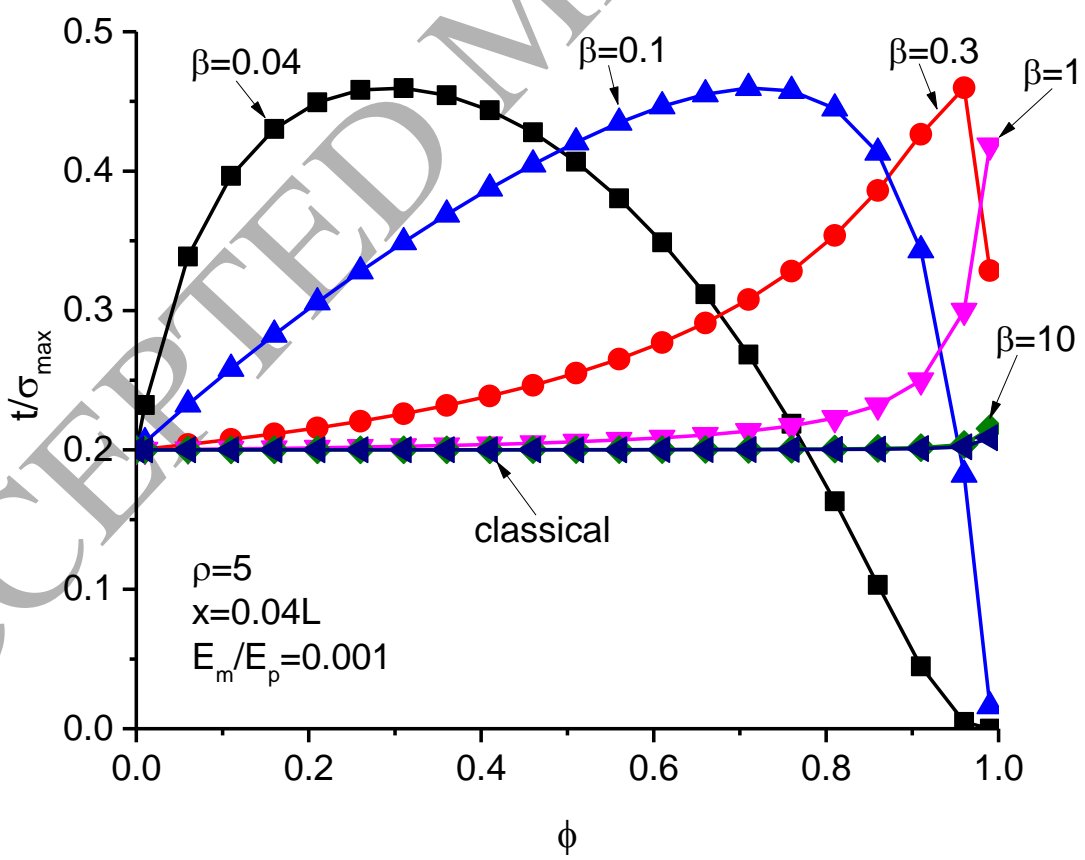

(c) 


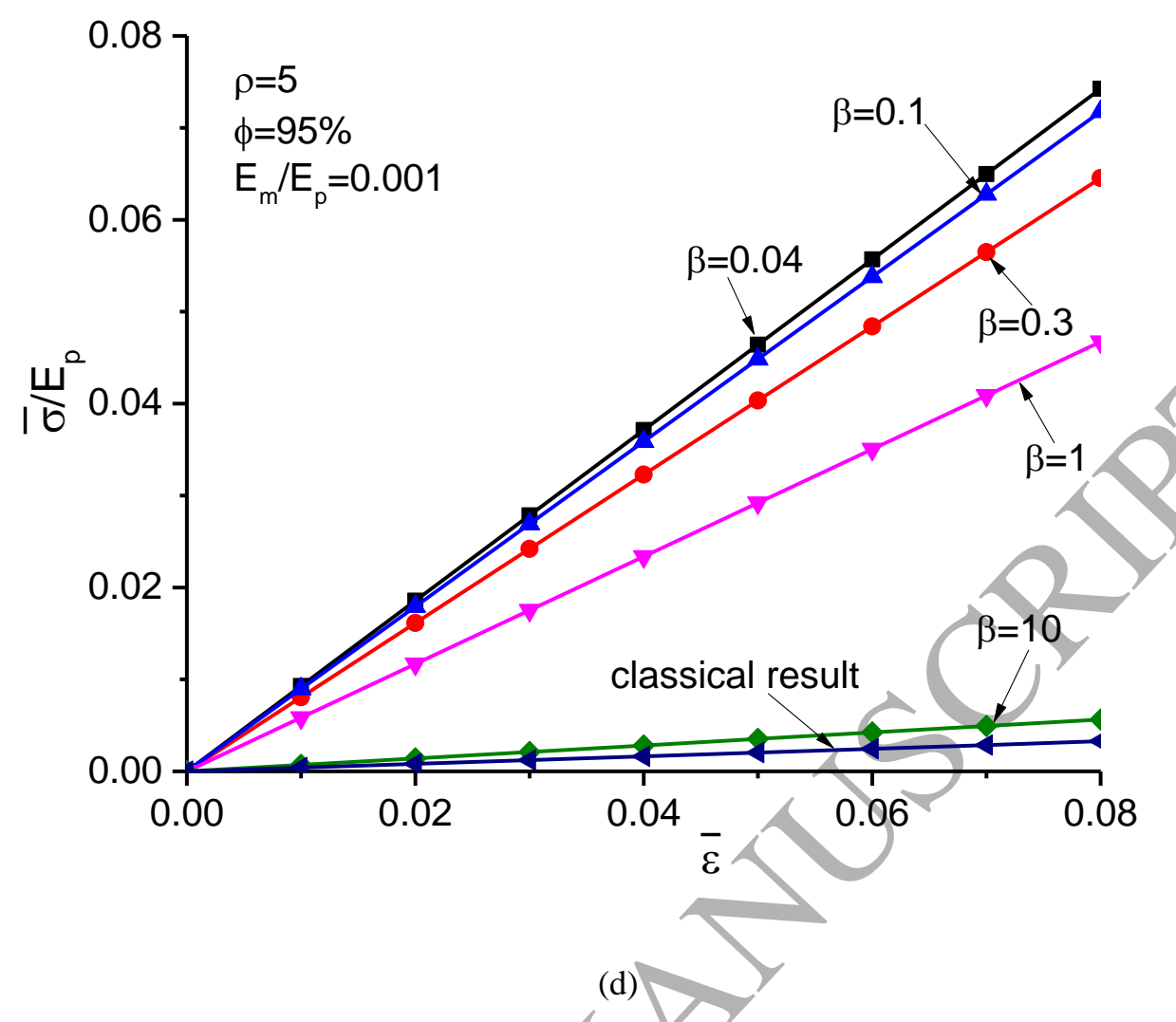

Fig.4 Variations of traction $t$ with $\mathrm{x}(\mathrm{a}), \rho$ (b), $\phi$ (c) for different $\beta$ and overall stress- strain relations (d) for different $\beta$

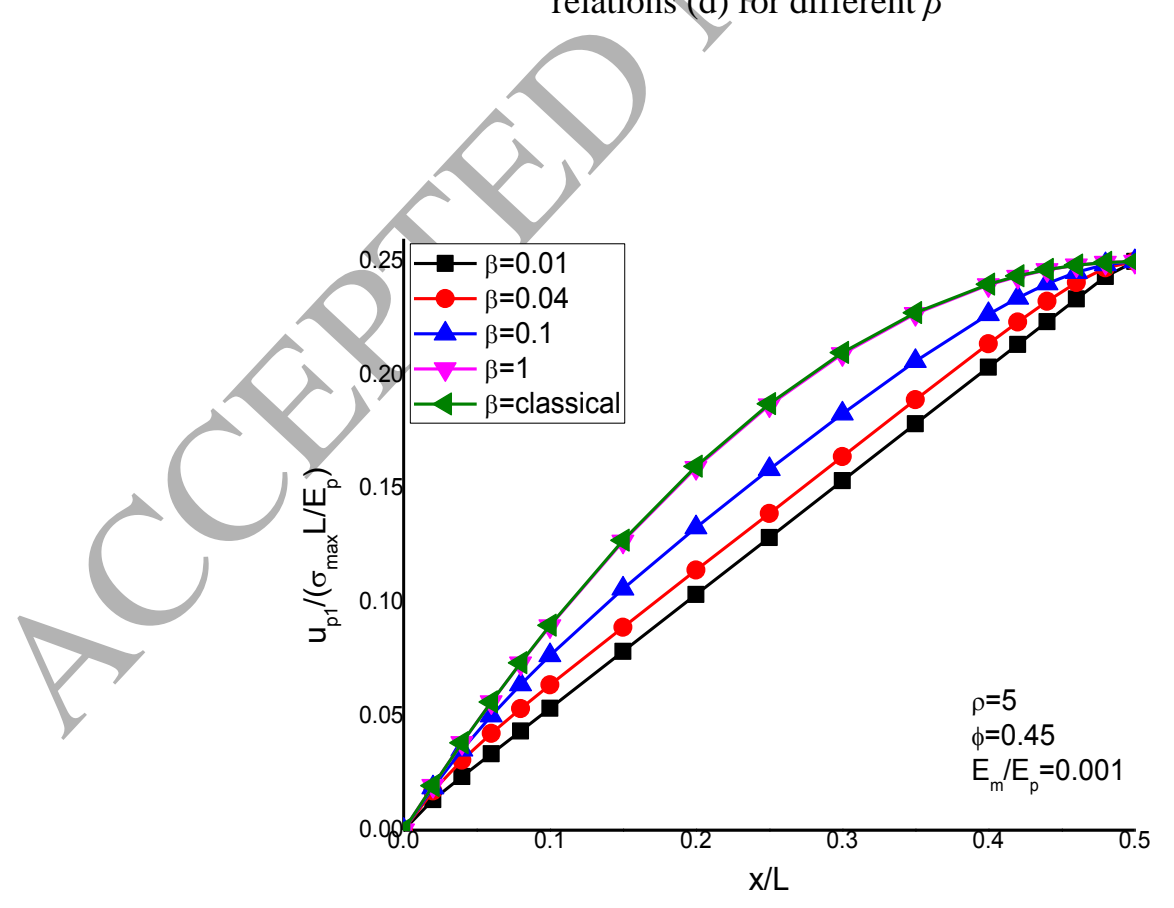

(a) 


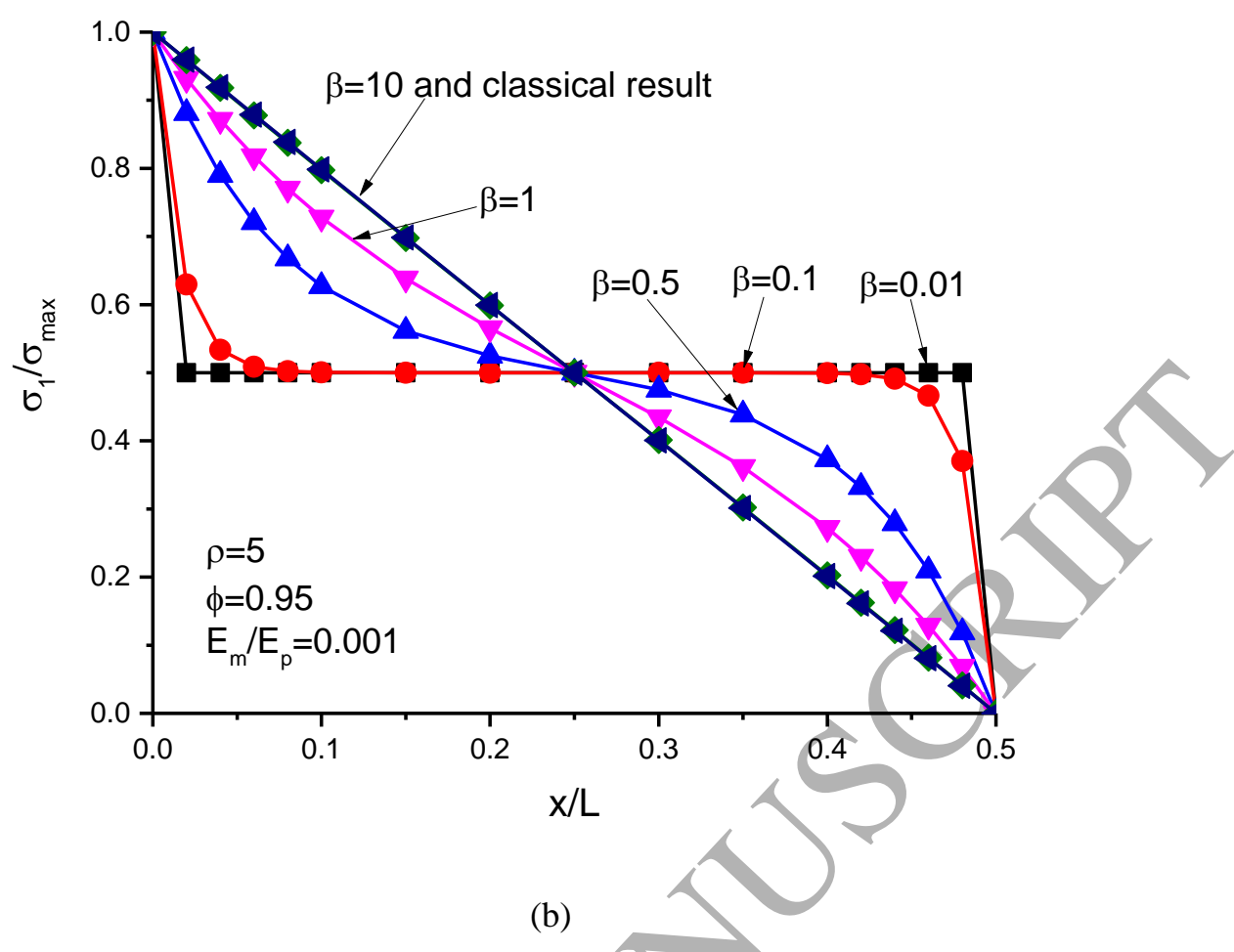

Fig.5. Distributions of displacement (a) and normal stress (b) along $x$ axis in the mineral platelet 1

\subsection{The effective modulus}

Predicted by Eqn.(2.17), the size effect of the effective Young's modulus $E_{c}$ of a staggered bio-structure will be examined in this subsection. The comparisons between the proposed model (abbreviated as SGSL) and some previously reported mechanical models will be carried out too.

The normalized effective modulus $E_{c} / E_{p}$ as a function of the modulus ratio of the organic matrix to mineral platelet $E_{m} / E_{p}$, the aspect ratio $\rho$ and the volume fraction $\phi$ are shown in Fig.6 (a), (b) and (c) respectively. We find that the effective modulus $E_{c} / E_{p}$ increases with the increases of $E_{m} / E_{p}, \rho$ or $\phi$.The smaller the $\beta$ value, the larger the $E_{c} / E_{p}$. Especially at small $E_{m} / E_{p}$ and small $\rho$, the modulus size effects are very big. That is to say, the smaller the thickness of the organic matrix corresponding to the smaller the value of $\beta$, the larger the effective 
modulus of the staggered structure is. On the other hand, when $\beta$ is large enough, the results should be degenerated into the classical ones.

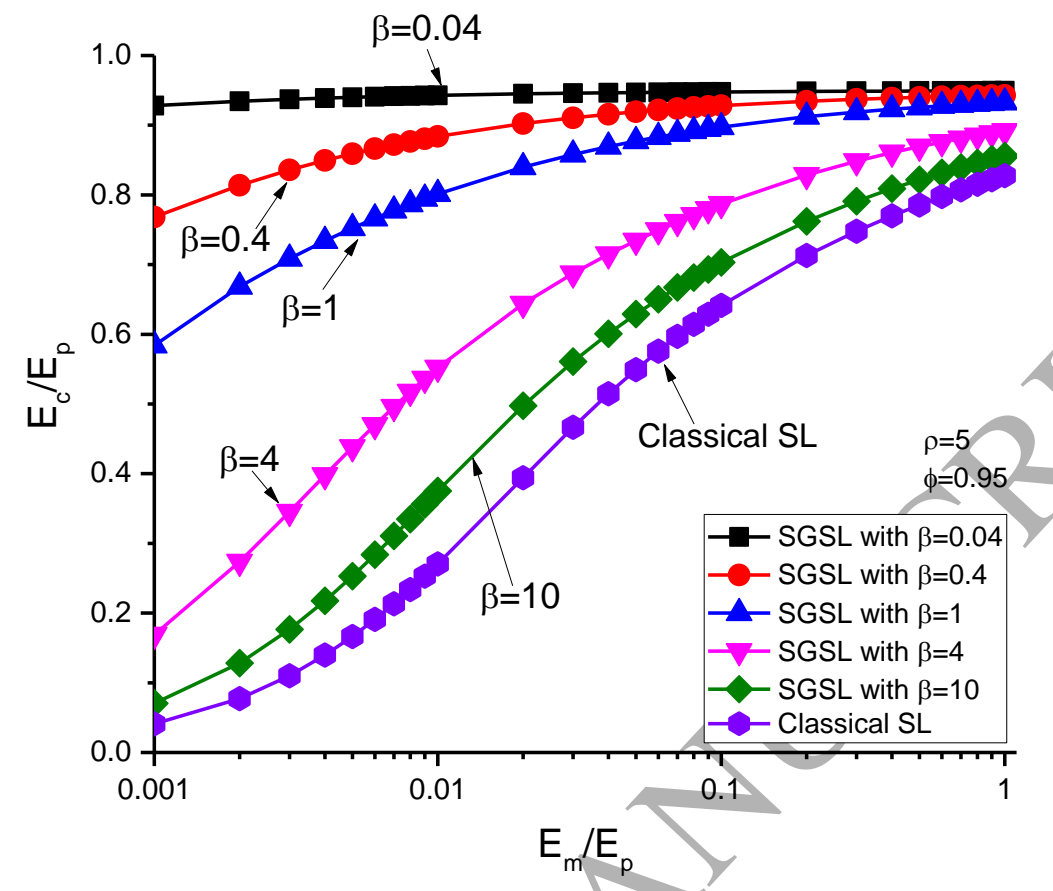

(a)

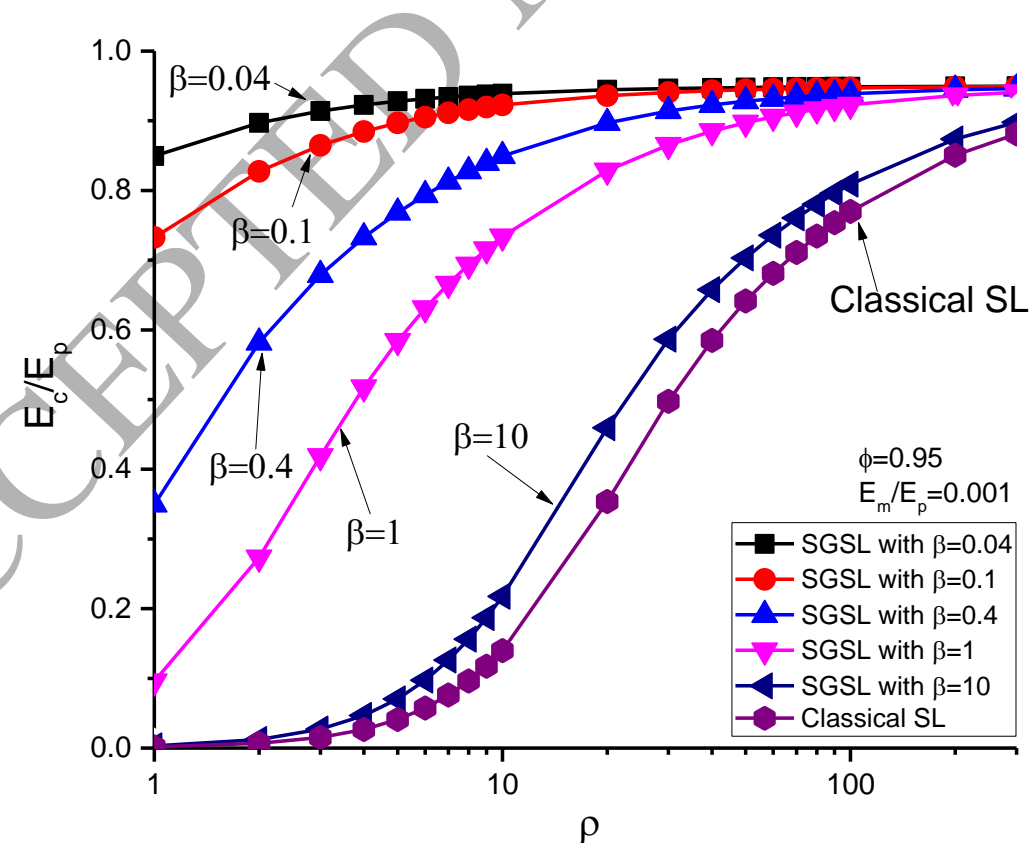

(b) 


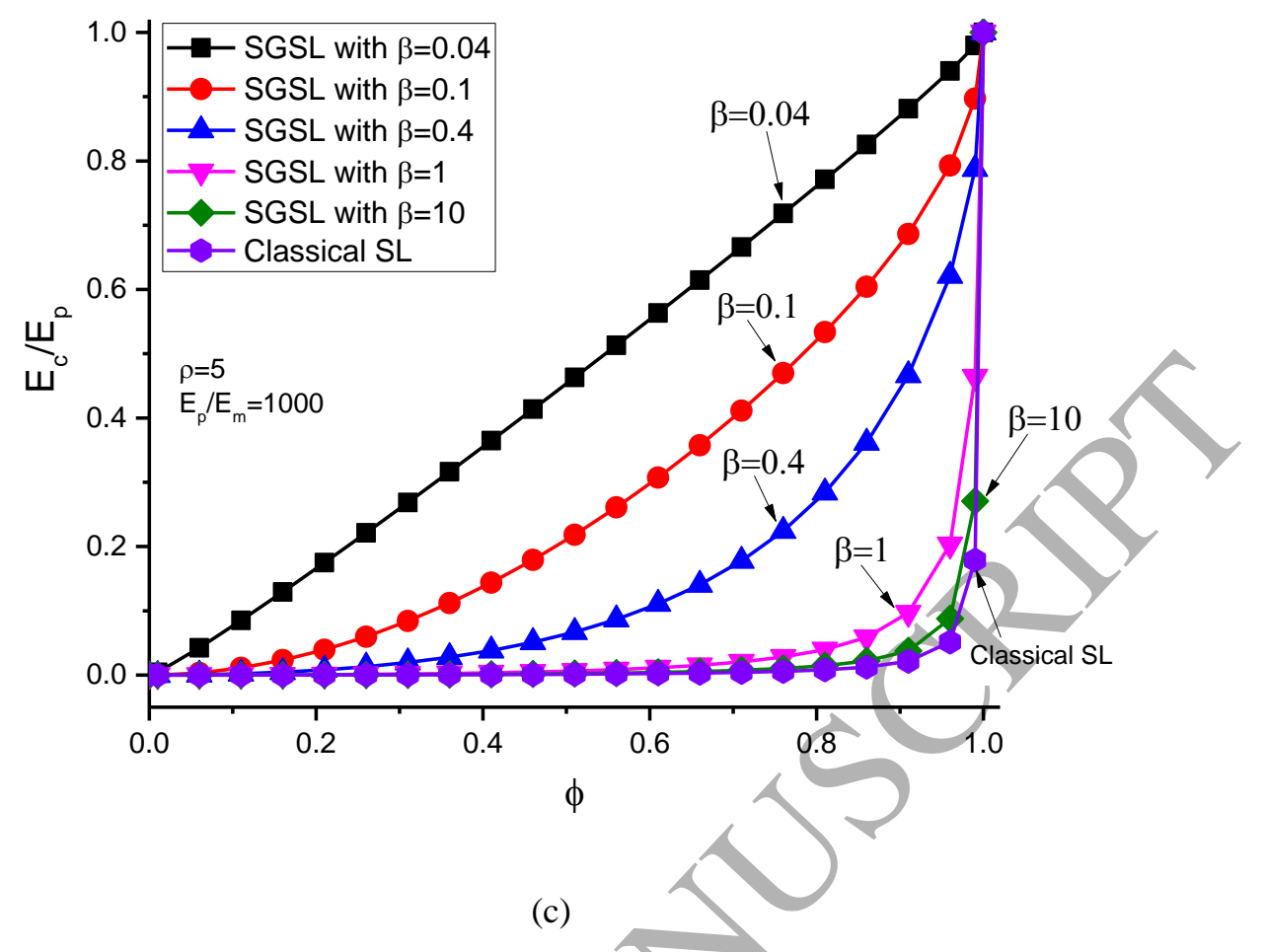

Fig.6. Variations of the effective modulus with $E_{m} / E_{p}$ (a), $\rho$ (b) and $\phi$ (c)

We also compare our results based on the strain gradient theory and shear-lag method (SGSL) with some previously reported results based on different models, such as Voigt's upper bound, Reuss lower bound, the classical shear-lag model (abbreviated as SL) (Zuo and Wei, 2007), Ji \& Gao's tension-shear-chain model (abbreviated as TSC) (Ji and Gao, 2004), Bar-on \& Wagner's model (abbreviated as BW)(Bar-On and Daniel Wagner, 2012) and Dai's model (abbreviated as Dai) (Dai et al., 2008), as shown in Fig.7.

The variation of effective modulus with modulus ratio $E_{m} / E_{p}$ is shown in Fig.7 (a) for both $\phi=95 \%$ and $\rho=10$, which are the features of nacre. We can find that for the real modulus ratio of organic materials, $E_{m} / E_{p}=0.001$, the present model can predict a more reasonable result than other models, which is closer to the modulus of mineral platelets. When the modulus ratio $E_{m} / E_{p}>0.1$, all models exceed the Voigt upper bound (Dai and BW) or Reuss lower bound (TSC, SL, SGSL) due to their respective assumptions, about which a lot of researches are still needed to be carried 
out. Fig.7 (b) shows the variations of the effective modulus with $\rho$ for $\phi=95 \%$, $E_{m} / E_{p}=0.001$. It implies that the present model has the wider applicable region for the aspect ratio $\rho$ than other model. For example, to obtain an exact effective modulus, such as $E_{c}=0.7 E_{p}$, the present model needs to set $\rho=7$ and other models need at least $\rho=40$ (Dai model). It means that our model is more suitable for a staggered structure with lower and more reasonable aspect ratio, such as for the case of nacre.

The effects of volume fraction $\phi$ of the mineral platelets on the effective modulus predicted by the above models are shown in Fig.7 (c) for $E_{m} / E_{p}=0.001$, $\rho=10$. The effective modulus increases gradually with $\phi \phi$ predicted by present model. However, other models predict very smaller $E_{c}$ and are insensitive to the variation of $\phi$ until $\phi>90 \%$, after which there are a drastic change of $E_{c}$. Therefore, for the case of $\phi<90 \%$ and small aspect ratio and small modulus ratio $E_{m} / E_{p}$, to use the present model can obtain the more reasonable predictions on the effective modulus of the staggered bio-structure than to use other models. 


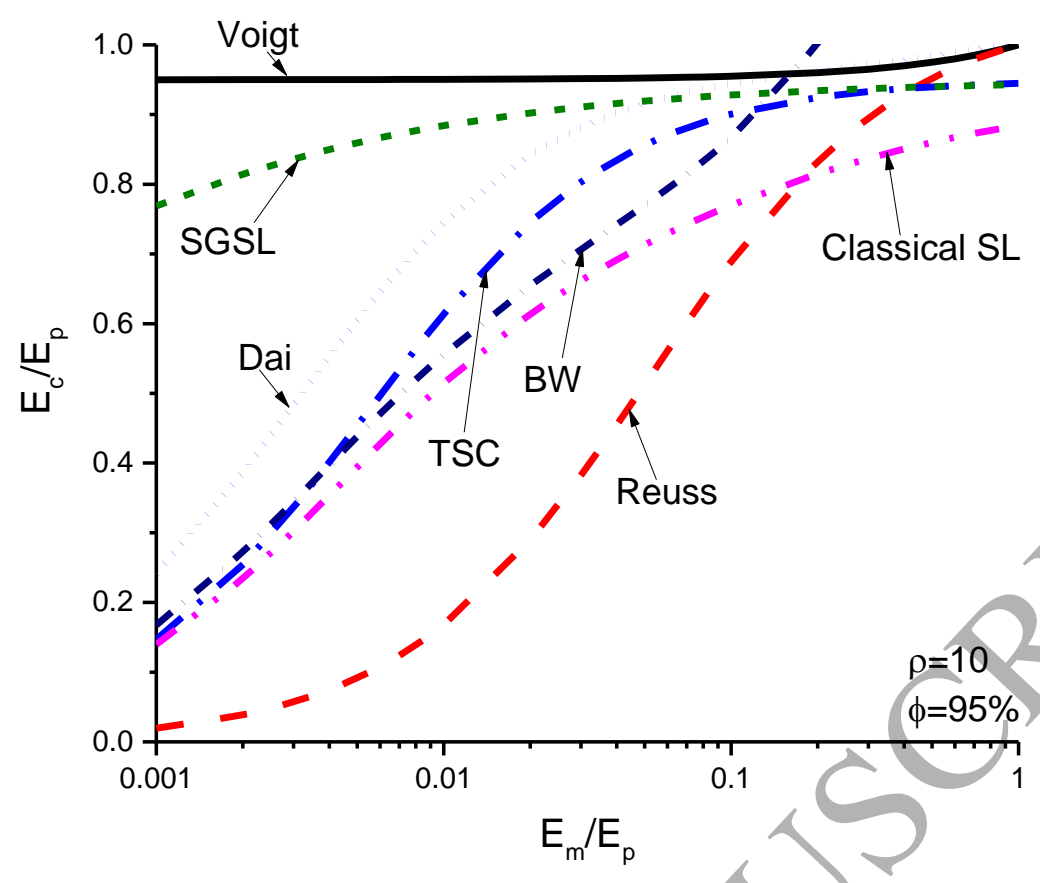

(a)

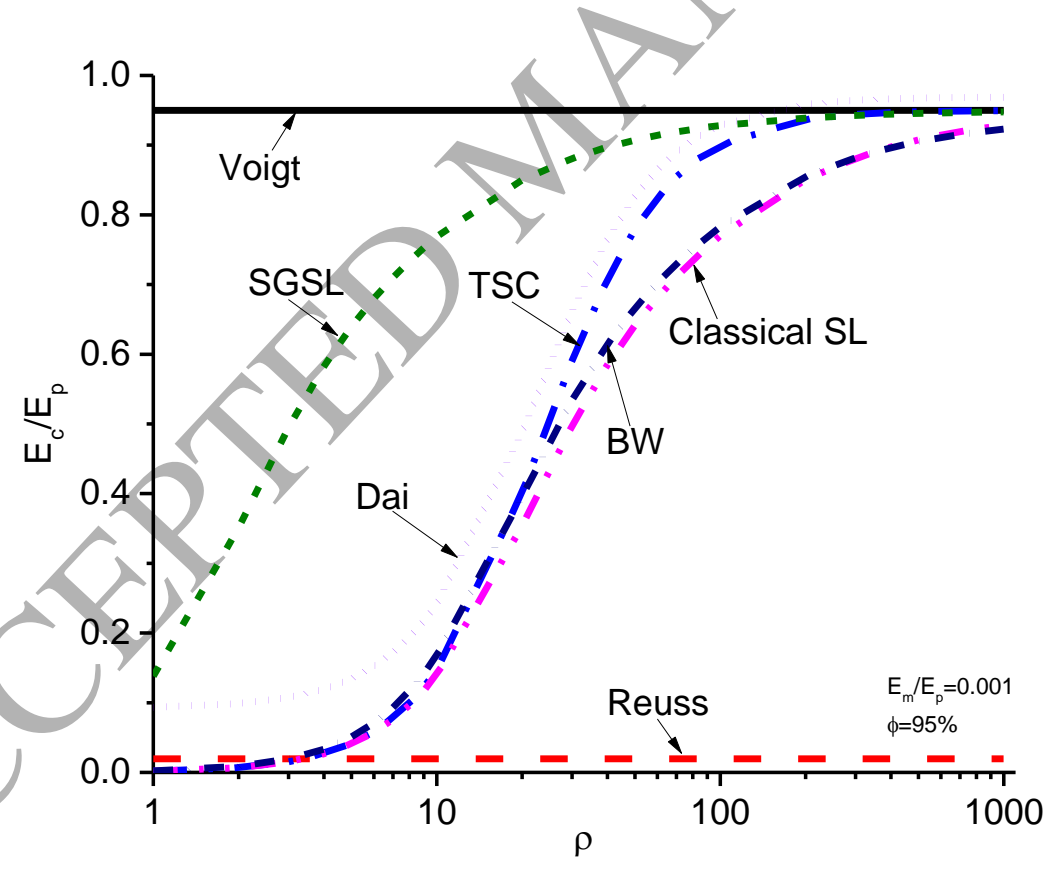

(b) 


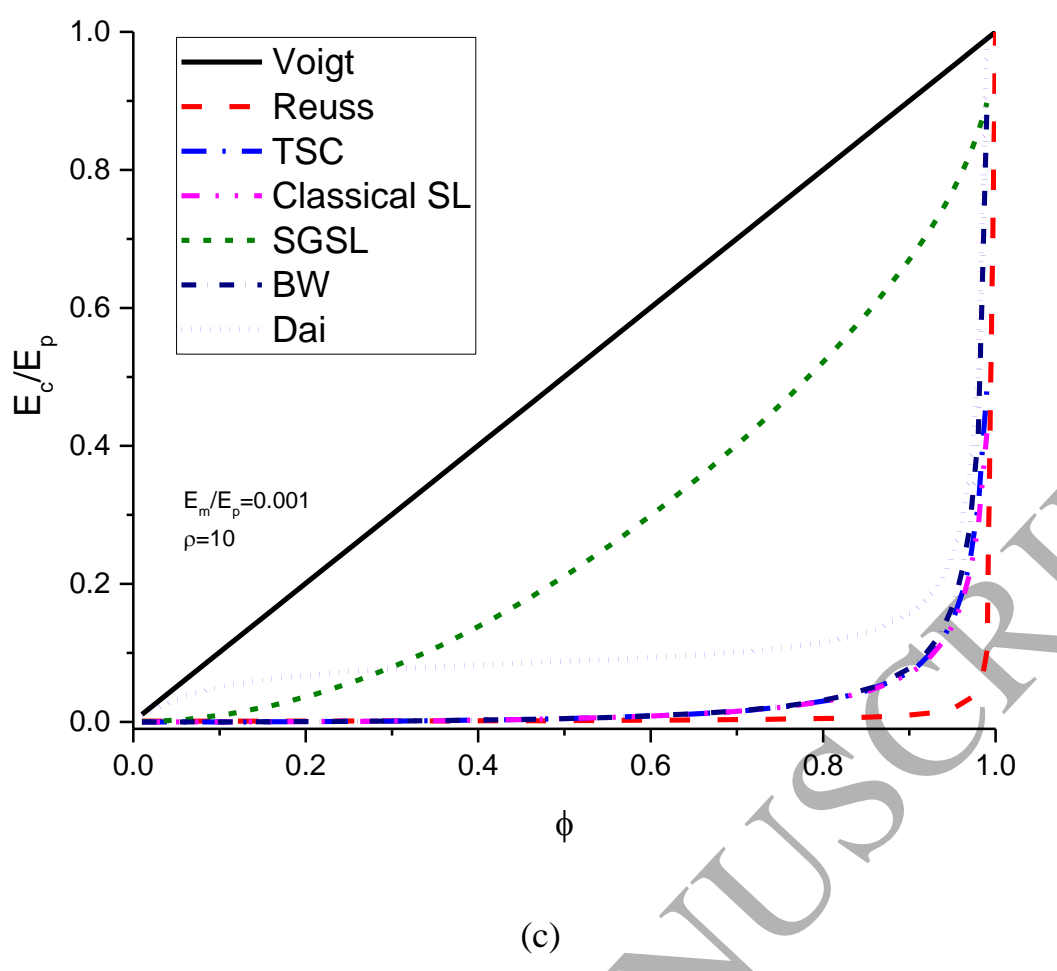

Fig.7. Comparisons of present model (SGSL) with other models for (a) $E_{c} / E_{p} \quad$ vs. $E_{m} / E_{p}$ (b)

$$
E_{c} / E_{p} \text { vs. } \rho \text { (c) } E_{c} / E_{p} \text { vs. } \phi
$$

\subsection{Comparisons with experiments and simulation}

Effective Young's modulus predicted by Eqn.(2.17) are compared with some experimental results (Barthelat et al., 2007; Fan, 2015; Jackson et al., 1988, 1986; Wang et al., 2001) and simulation (Katti et al., 2001) for nacre. The characteristic length of the strain gradient organic matrix is an essential parameter in the proposed model. For metal, this characteristic length is in the order of micron, however for organic material (nacre), its value is also in the order of micron through comparing simulation results with experimental results for nanoindentation test (Song et al., 2015). So, in the following calculations, we will use the values of characteristic length for the organic matrix.

Fan (2015) focused on Hyriopsis cumingii in his master thesis. He found that the length and thickness of the mineral platelets were about $L=4.6 \mu m$ and $h=0.93 \mu m$ respectively, the thickness of the organic matrix was $d=20 \mathrm{~nm}$. The volume fraction 
of the organic matrix was $14 \%$. But there was only $2.1 \%(\approx d /(d+h))$ in the interlaminations, the rest $11.9 \%$ was in the mineral platelets, which divided the mineral platelet to many aragonite nanograins (Zhang et al., 2016). So we derived the modulus of the mineral platelet is $E_{p} \approx 94 \mathrm{GPa}$ computed simply by the Voigt model (see Appendix C). The nanoidentation test was carried out on the nacre layer of Hyriopsis cumingii. The measured Young's modulus was $87.0 \pm 3.9 \mathrm{GPa}$ and the hardness showed a strong size effect. By a trans-scale mechanics theory (Song et al., 2014; Wu et al., 2012), which considered both strain gradient effect and the surface/interface effect, Song et al. (2015) simulated this size effect of hardness and gave the characteristic length of the strain gradient of the nacre as $l=0.9 \mu m$ or $l=2.3 \mu \mathrm{m}$. So the two characteristic lengths will be used as the characteristic length of the strain gradient organic matrix approximately in the calculation for Hyriopsis cumingii. According to Eqn.(2.17), the effective modulus of nacre in Hyriopsis cumingii is $91.4 \mathrm{GPa}(l=0.9 \mu \mathrm{m})$ or $91.9 \mathrm{GPa}(l=2.3 \mu \mathrm{m})$. It is coincided well with the experimental result of $87.0 \pm 3.9 \mathrm{GPa}$. And therefore, $l=0.9 \mu \mathrm{m}$ will be used approximately as the characteristic length of organic matrix in the following calculations.

As mentioned above, the organic materials and the immersed mineral bridges, nanoasperities, etc. in the organic interlaminations can be regarded as an effective medium and its properties are size-dependence. This size effect is relevant to the size of mineral bridges or nanoasperities and the thickness of the organic layers.

The geometry parameters used in each calculation are selected from the corresponding experiments and simulation and they are listed in Table 1. The Young's moduli of the mineral platelets and organic matrix are set to be $E_{p}=94 \mathrm{GPa}$ and $E_{m}=0.1 \mathrm{GPa}$ for the cases in which these material parameters are not given explicitly. With the help of Table 1 and Eqn.(2.17), the effective moduli of nacreous layer in different shells with characteristic lengths of $l=0.9 \mu m$ are evaluated and listed in Table 2, as well as the results evaluated by TSC model (Ji and Gao, 2004), 
SL model (Zuo and Wei, 2007), BW model (Bar-On and Daniel Wagner, 2012) and Dai model (Dai et al., 2008). We can conclude that our present model gives the best predictions for the effective modulus of nacre compared to the experimental and simulation results.

Table 1 the material parameters selected from experiments and simulation on nacre.

\begin{tabular}{|c|c|c|c|c|c|c|c|c|}
\hline & Shell & $\begin{array}{c}L \\
(\mu \mathrm{m})\end{array}$ & $\begin{array}{c}h \\
(\mu \mathrm{m})\end{array}$ & $\begin{array}{c}d \\
(\mathrm{~nm})\end{array}$ & $\begin{array}{c}E_{p} \\
(\mathrm{GPa})\end{array}$ & $\begin{array}{r}E_{m} \\
(\mathrm{GPa})\end{array}$ & $\rho=\frac{L}{h}$ & $=\frac{h}{d+h}$ \\
\hline $\operatorname{Fan}(2015)$ & $\begin{array}{l}\text { Hyriopsis } \\
\text { cumingii }\end{array}$ & 4.6 & 0.93 & 20 & 94 & 1 & & \\
\hline $\begin{array}{l}\text { Barthelat } \\
\text { ( 2007) }\end{array}$ & $\begin{array}{c}\text { Red } \\
\text { abalone }\end{array}$ & 8 & 0.4 & 20 & & & 20 & $95 \%$ \\
\hline $\begin{array}{l}\text { Katti (2001) } \\
\text { (simulation) }\end{array}$ & 1 & 5.6 & 0.5 & 30 & & 0.02 & 11 & $94 \%$ \\
\hline Wang (2001) & Red abalone & 6.5 & 0.55 & & 1 & 1 & 12 & $96 \%$ \\
\hline $\begin{array}{c}\text { Jackson(1988, } \\
1986)\end{array}$ & Pinctada & 4 & & 15 & 1 & 1 & 10 & $96 \%$ \\
\hline
\end{tabular}

Table 2. The effective Young's modulus (GPa) for nacre.

\begin{tabular}{|c|c|c|c|c|c|}
\hline & Fan(2015) & $\begin{array}{c}\text { Barthelat } \\
(2007)\end{array}$ & $\begin{array}{c}\text { Katti (2001) } \\
(\text { simulation })\end{array}$ & Wang( 2001) & $\begin{array}{c}\text { Jackson } \\
(1988,1986)\end{array}$ \\
\hline $\begin{array}{c}\text { Experim } \\
\text { ents }\end{array}$ & $\begin{array}{c}87.0 \pm 3.9 \\
\text { Present }\end{array}$ & 90 & $20-25$ & $69 \pm 7$ & $70 \pm 11$ \\
\hline model & $91.4(l=0.9 \mu \mathrm{m})$ & $89(l=0.9 \mu \mathrm{m})$ & $91.5(l=0.9 \mu \mathrm{m})$ & $89.8(l=0.9 \mu \mathrm{m})$ & $89.9(l=0.9 \mu \mathrm{m})$ \\
\hline TSC & 10.26 & 40.1 & 3.31 & 23.9 & 18 \\
\hline SL & 9.89 & 35.4 & 3.26 & 22.1 & 17 \\
\hline BW & 9.3 & 36.4 & 3.6 & 23.5 & 18.2 \\
\hline Dai & 17.1 & 44.9 & 5.5 & 31.3 & 25.8 \\
\hline
\end{tabular}


It is worth noting that according to studies of Gao et al.(2003), Ji and Gao (2004) et al., the cases of the bone and teeth are mainly different from nacre in their aspect ratio of the mineral platelets (ratio of the length to thickness). The aspect ratios of the bone and teeth are about 30 60, however the aspect ratio of the nacre is about 5 20. Additionally, the thicknesses of the mineral platelets for bone and teeth are about several to tens nanometers, while the thickness of the nacre is about one micron. So in the present paper we presented the model based on the strain gradient theory to analyze the mechanical behave for the nacre. Theoretically, the model is applicable to the broad cases, such as bone and teeth. Ji and Gao (2004) stressed that the predicted results based on the tension-shear chain model are suitable for a mineral platelet of aspect ratio larger than 30 . We shall use the model to describe a broad case in our future studies.

\section{Conclusions}

Based on the strain gradient theory, a trans-scale shear-lag model is developed to characterize the mechanical properties of staggered bio-structure. In the proposed model, the organic materials in the interlaminations are considered as elastic strain gradient continuum and the mineral platelets are still classical elastic materials, then the classical shear-lag model is extended to strain gradient continuum case. The fundamental elastic fields and the analytical expression of the effective modulus of the staggered bio-structure are obtained. The results show that the mechanical behavior of the staggered bio-structure materials has the strong size effects. A parameter $\beta=\frac{d}{l}$ is proposed to characterize the size effects. The results show that the thinner the organic matrix layer, corresponding to the smaller $\beta$ value, the greater the size effect of mechanical behavior. When the thickness of the organic matrix layer is large enough or $\beta$ is large enough, the size effect is small enough to be neglected, and then the proposed model returns to the classical shear-lag model. The model incorporates the effects of microstructure and scale, and thus it is more suitable for the 
staggered bio-structure with small aspect ratio and small modulus ratio of organic matrix to mineral platelets. The predicted effective moduli of nacreous layer in different shells are consistent well with the corresponding experimental results.

\section{Acknowledgments}

This work is supported by the National Natural Science Foundation of China under Grants No. 11572329, 11372318, 11672296, 11432014, 11672301, 11521202 and by the Strategic Priority Research Program of the Chinese Academy of Sciences, Grant No. XDB22040501.

\section{References}

Bar-On, B., Daniel Wagner, H., 2012. Elastic modulus of hard tissues. J. Biomech. 45, 672-678. doi:10.1016/j.jbiomech.2011.12.003

Bar-On, B., Wagner, H.D., 2011. Mechanical model for staggered bio-structure. J. Mech. Phys. Solids 59, 1685-1701. doi:10.1016/j.jmps.2011.06.005

Barthelat, F., 2007. Biomimetics for next generation materials. Philos.Trans. R. Soc. London, Ser. A 365, 1861.

Barthelat, F., Tang, H., Zavattieri, P.D., Li, C.M., Espinosa, H.D., 2007. On the mechanics of mother-of-pearl: A key feature in the material hierarchical structure. J. Mech. Phys. Solids 55, 306-337. doi:10.1016/j.jmps.2006.07.007

Currey, J.D., 1977. Méchanical properties of mother of pearl in tension. Proc. R. Soc. Lond. B, 196, 443-463.

Dai, Y., Mai, Y.W., Ji, X., 2008. Predictions of stiffness and strength of nylon 6/MMT nanocomposites with an improved staggered model. Compos. Part B Eng. 39, 1062-1068. doi:10.1016/j.compositesb.2007.09.005

Dunlop, J.W.C., Fratzl, P., 2010. Biological Composites. Annu. Rev. Mater. Res. 40, 1-24. doi:10.1146/annurev-matsci-070909-104421

Espinosa, H.D., Rim, J.E., Barthelat, F., Buehler, M.J., 2009. Merger of structure and material in nacre and bone - perspectives on de novo biomimetic materials. Prog. Mater. Sci. 54, 8. 
Fan, C., 2015. Experimental inverstigation of the hierarchical structure and mechanical properties of molluscan shells. Institue of Mechanics, Chinese Academy of Science,Beijing.

Fleck, N.A., Hutchinson, J.W., 1993. A phenomenological theory for strain gradient effects in plasticity. J. Mech. Phys. Solids 41, 1825-1857.

Gao, H., 2006. Application of fracture mechanics concepts to hierarchical biomechanics of bone and bone-like materials. Int. J. Fract. 138, 101-137.

Gao, H., Ji, B., Jager, I.L., Arzt, E., Fratzl, P., 2003. Materials become insensitive to flaws at nanoscale: Lessons from nature. Proc. Natl. Acad. Sci. 100, 5597-5600. doi:10.1073/pnas.0631609100

Jackson, A.P., Vincent, J.F. V., Briggs, D., Crick, R.A., Davies, S.F., Hearn, M.J., Turner, R.M., 1986. Application of surface analytical techniques to the study of fracture surfaces of mother-of-pearl. J. Mater. Sci. Lett. 5, 975-978. doi:10.1007/BF01730253

Jackson, A.P., Vincent, J.F. V., Turner, R.M., 1988. The Mechanical Design of Nacre. Proc. R. Soc. B Biol. Sci. 234, 415-440. doi:10.1098/rspb.1988.0056

Jäger, I., Fratzl, P., 2000. Mineralized Collagen Fibrils: A Mechanical Model with a Staggered Arrangement of Mineral Particles. Biophys. J. 79, 1737-1746. doi:10.1016/S0006-3495(00)76426-5

Ji, B., Gao, H., 2010. Mechanical Principles of Biological Nanocomposites. Annu. Rev. Mater.Res, 40, 77-100. doi:10.1146/annurev-matsci-070909-104424

Ji, B., Gao, H., 2004. Mechanical properties of nanostructure of biological materials. J. Mech. Phys. Solids 52, 1963-1990. doi:10.1016/j.jmps.2004.03.006

Katti, D.., Katti, K.., Sopp, J.., Sarikaya, M., 2001. 3D finite element modeling of mechanical response in nacre-based hybrid nanocomposites. Comput. Theor. Polym. Sci. 11, 397-404. doi:10.1016/S1089-3156(01)00012-5

Kinney, J.H., Marshall, S.J., Marshall, G.W., 2003. The mechanical properties of human dentin: a critical review and re-evaluation of the dental literature. Crit. Rev. Oral Biol. Med. 14, 13-29.

Landis, W.J., 1995. The strength of a calcified tissue depends in part on the molecular 
structure and organization of its constituent mineral crystals in their organic matrix. Bone 16, 533-544.

Marshall, G.W.J., Marshall, S.J., Kinney, J.H., Balooch, M., 1997. The dentine substrate: structure and properties related to bonding. J. Dent. 25, 441-458.

Menig, R., Meyers, M.H., Meyers, M.A., Vecchio, K.S., 2000. Quasi-static and dynamic mechanical response of Haliotis rufescens (abalone) shells. Acta Mater. 48, 2383-2398.

Meyers, M.A., Chen, P.Y., Lin, A.Y.M., Seki, Y., 2008. Biological materials: Structure and mechanical properties. Prog. Mater. Sci. 53, 1-206. doi:10.1016/j.pmatsci.2007.05.002

Song, F., Soh, A.K., Bai, Y.L., 2003. Structural and mechanical properties of the organic matrix layers of nacre. Biomaterials 24, 3623-3631. doi:10.1016/S0142-9612(03)00215-1

Song, J., Fan, C., Ma, H., Wei, Y., 2015. Hierarchical structure observation and nanoindentation size effect characterization for a limnetic shell. Acta Mech. Sin. 31, 364-372. doi:10.1007/s10409-015-0405-X

Song, J., Liu, J., Ma, H., Liang, L., Wei, Y., 2014. Determinations of both length scale and surface elastic parameters for fcc metals. Comptes Rendus - Mec. 342, 315-325. doi:10.1016/j.crme.2014.03.004

Sun, J., Bhushan, B., 2012. Hierarchical structure and mechanical properties of nacre: a review. RSC Adv. 2, 7617. doi:10.1039/c2ra20218b

Tesch, W., Eidelman, N., Roschger, P., Goldenberg, F., Klaushofer, K., Fratzl, P., 2001. Graded microstructure and mechanical properties of human crown dentin. Calcif.Tissue Int. 69, 147-157.

Wang, R.Z., Suo, Z., Evans, A.G., Yao, N., Aksay, I.A., 2001. Deformation mechanisms in nacre. J. Mater. Res. 16, 2485-2493. doi:10.1557/JMR.2001.0340

Wegst, U., Ashby, M., 2004. The mechanical efficiency of natural materials. Philos. Mag. 84, 2167-81.

Wei, Y., 2006. A new finite element method for strain gradient theories and 
applications to fracture analyses. Eur. J. Mech. A/Solids 25, 897-913.

doi:10.1016/j.euromechsol.2006.03.001

Wei, Y., Hutchinson, J., 1997. Steady-state crack growth and work of fracture for solids characterized by strain gradient plasticity. J. Mech. Phys. Solids 45, 12531273.

Weiner, S., Wagner, H.D., 1998. The material bone:structure-mechanical function relations. Annu. Rev. Mater. Res. 28, 271.

Wu, B., Liang, L.H., Ma, H.S., Wei, Y.G., 2012. A trans-scale model for size effects and intergranular fracture in nanocrystalline and ultra-fine polycrystalline metals. Comput. Mater. Sci. 57, 2-7.

Zhang, N., Yang, S., Xiong, L., Hong, Y., Chen, Y., 2016. Nanoscale toughening mechanism of nacre tablet. J. Mech. Behav. Biomed. Mater. 53, 200-209. doi:10.1016/j.jmbbm.2015.08.020

Zhang, Z.Q., Liu, B., Huang, Y., Hwang, K.C., Gao, H., 2010. Mechanical properties of unidirectional nanocomposites with non-uniformly or randomly staggered platelet distribution. J. Mech. Phys. Solids 58, 1646-1660. doi:10.1016/j.jmps.2010.07.004

Zuo, S., Wei, Y., 2007. Effective elastic modulus of bone-like hierarchical materials. Acta Mech. Solida Sin. 20, 198-205. doi:10.1007/s10338-007-0723-z

\section{Appendices:}

\section{A. the expressions of stress fields in the staggered structure}

The normal stress in the mineral platelets:

$$
\begin{aligned}
& \sigma_{1}(x)=\frac{\sigma_{\max }}{2}\left[1-\frac{1}{e^{\eta / 2}-1} e^{\eta x / L}+\frac{e^{\eta / 2}}{e^{\eta / 2}-1} e^{-\eta x / L}\right] \\
& \sigma_{2}(x)=\frac{\sigma_{\max }}{2}\left[1+\frac{1}{e^{\eta / 2}-1} e^{\eta x / L}-\frac{e^{\eta / 2}}{e^{\eta / 2}-1} e^{-\eta x / L}\right]
\end{aligned}
$$

the shear stress and the high order stress in the strain gradient organic matrix

$$
\sigma_{21}(x, y)=\frac{G_{m} L \sigma_{\max }}{E_{p} \eta} \frac{A\left[1-e^{A y}+e^{A d}\left(1-e^{-A y}\right)\right]}{2\left(1-e^{A d}\right)+A d\left(1+e^{A d}\right)}\left[\frac{1}{e^{\eta / 2}-1} e^{\eta x / L}+\frac{e^{\eta / 2}}{e^{\eta / 2}-1} e^{-\eta x / L}\right]
$$




$$
\tau_{221}(x, y)=\frac{2 E_{m} l^{2} L \sigma_{\max }}{\eta E_{p}} \frac{A^{2}\left(e^{A d} e^{-A y}-e^{A y}\right)}{2\left(1-e^{A d}\right)+A d\left(1+e^{A d}\right)}\left[\frac{1}{e^{\eta / 2}-1} e^{\eta x / L}+\frac{e^{\eta / 2}}{e^{\eta / 2}-1} e^{-\eta x / L}\right]
$$

\section{B. The effective modulus expressions for a staggered structure of TSC, SL, Dai and BW models}

The sizes of the mineral platelets and the organic matrix are defined the same as section 2 and the gap between the ends of two mineral platelets is defined as $a$ additionally. For TSC, SL and our SGSL models, the gap $a$ is assumed small enough to be neglected such that the volume fraction of mineral platelets is $\phi_{1}=\frac{h}{h+d}$. But for Dai and BW models, the gap $a$ is considered so that the definition of volume fraction is $\phi_{2}=\frac{L \times h}{(a+L)(d+h)} . E_{p}$ is the Young's modulus of mineral platelets and $E_{m}, G_{m}$ are the Young's and shear moduli of organic matrix respectively. The aspect ratio of the mineral platelets is $\rho=L / h$.

The effective modulus expressions are:

1) Tension-shear-chain (TSC) model (Gao et al., 2003; Ji and Gao, 2004)

$$
\frac{1}{E_{T S C}}=\frac{1}{\phi_{1} E_{p}}+\frac{16}{\eta_{c}^{2} \phi_{1} E_{p}}
$$

where $\eta_{c}=2 \rho \sqrt{\frac{G_{m} \phi_{1}}{E_{p}\left(1-\phi_{1}\right)}}$.

2) Shear-lag (SL) model (Zuo and Wei, 2007)

$$
\frac{1}{E_{S L}}=\frac{1}{\phi_{1} E_{p}}+\frac{4}{\eta_{c} \phi_{1} E_{p} \tanh \left(\frac{\eta_{c}}{4}\right)}
$$

where $\eta_{c}=2 \rho \sqrt{\frac{G_{m} \phi}{E_{p}(1-\phi)}}$ is the same as that in TSC model.

3) Dai's model (Dai et al., 2008)

$$
E_{D a i}=\frac{a+L}{2(d+h) \times \omega} \times \frac{\frac{2}{K_{B}}+\frac{1}{K_{c}}+\frac{1}{K_{D}}}{1+C_{M N}}
$$


where $K_{A}=\frac{L}{2 E_{p} \times h \times \omega}, \quad K_{B}=\frac{a}{E_{m} \times h \times \omega}, \quad K_{C}=\frac{2 d}{G_{m} \times L \times \omega}, \quad K_{D}=\frac{a}{2 E_{m} \times d \times \omega}$

$$
K_{C D}=\frac{L}{2 E_{m} \times d \times \omega}, C_{M N}=\frac{\frac{2}{K_{B}}+\frac{1}{K_{C}}+\frac{1}{K_{D}}}{\frac{1}{K_{A}}+\frac{1}{K_{C D}}}
$$

where $\omega$ is the out-of-plane thickness of the staggered structure.

4) BW model (Bar-On and Daniel Wagner, 2012)

$$
E_{B W}=\frac{\gamma}{\left(2 \frac{\phi_{2}}{\Delta_{p}}-1\right) \operatorname{ctgh}\left(\frac{\gamma}{2}\right)+\gamma\left(\frac{3}{2}-\frac{\phi_{2}}{\Delta_{p}}\right)} \bar{E}
$$

where

$$
\begin{aligned}
& \bar{E}=\frac{1}{2}\left[E_{p} \Delta_{p}+E_{m}\left(2-\Delta_{p}\right)\right], \quad \gamma^{2}=4 \rho^{2} \frac{G_{m}}{E_{p}} \frac{\Delta_{p}}{1-\Delta_{p}}\left(1-\frac{\Delta_{p}}{2 \phi_{2}}\right)^{2} \\
& \Delta_{p}=\frac{h}{h+d}, \quad \phi_{2}=\frac{L}{L+a} \Delta_{p},
\end{aligned}
$$

To compare with our model, the gap $a$ is set to be $a=0.01 L$ for Dai and BW models in calculations. For calculating their curves presented in Fig.10, $L=\rho \times h$, $\phi_{2}=\frac{L \times h}{(a+L)(d+h)}$ are used to derive the relations between the effective modulus and the aspect ratio and volume fraction of mineral platelets. For calculating their data presented in Table 2, the measured $L, h, d$ listed in Table 1 are used.

\section{The effective modulus of the mineral platelets of Hyriopsis cumingii based on the experiment performed by Fan(2015)}

In the nacreous layer of Hyriopsis cumingii, Fan (2015) found that the volume fraction of the organic matrix was $14 \%$. But there was only $2.1 \%\left(\approx \frac{d}{d+h}\right)$ in the interlaminations, the rest $11.9 \%$ was in the mineral platelets. So the effective modulus of the mineral platelet can be computed simply by Voigt model. That is,

$$
E_{p}=E_{\text {aragonite }} f_{\text {nanograin }}+\left(1-f_{\text {nanograin }}\right) E_{m}
$$


where

$$
f_{\text {nanograin }}=\frac{86 \%}{86 \%+11.9 \%}=87.8 \%, E_{\text {aragonite }}=107 \mathrm{GPa}(\text { Fan }, 2015), E_{m}=100 \mathrm{MPa} .
$$

Then $E_{p} \approx 94 \mathrm{GPa}$.

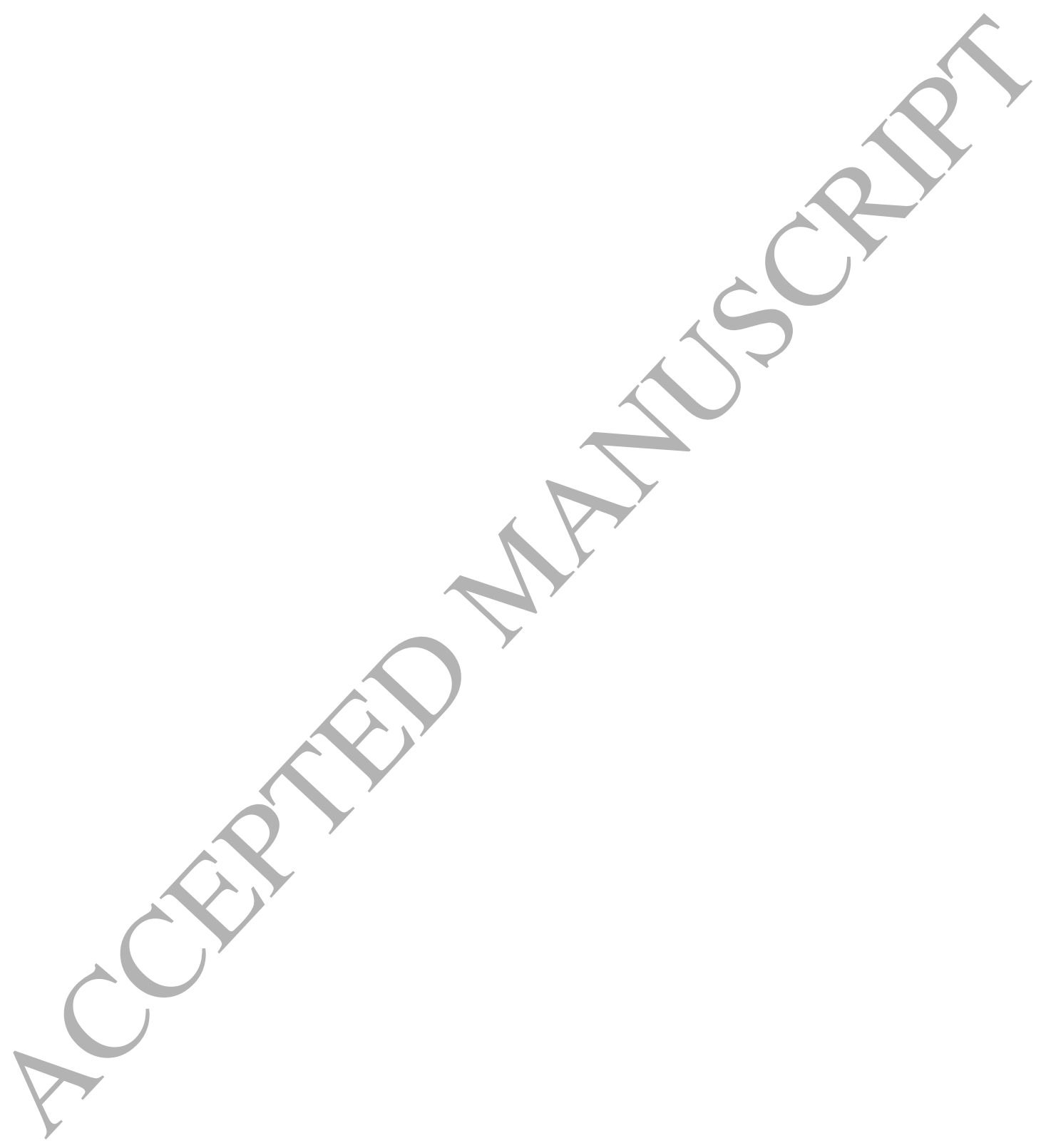


graphical abstract

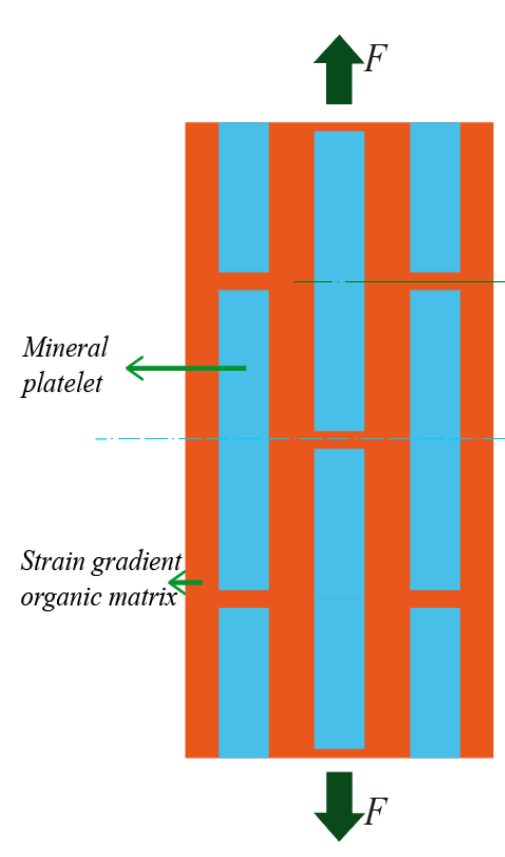

(a)

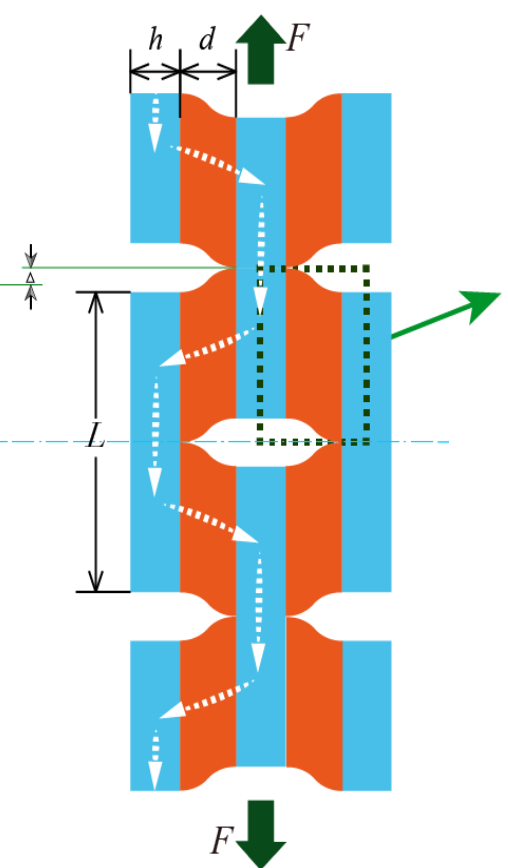

(b)

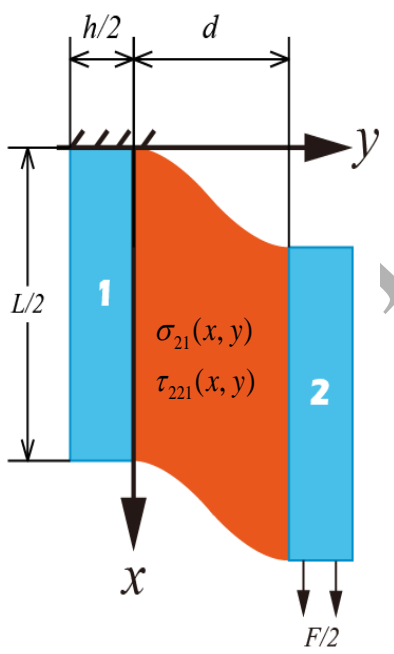

(c)

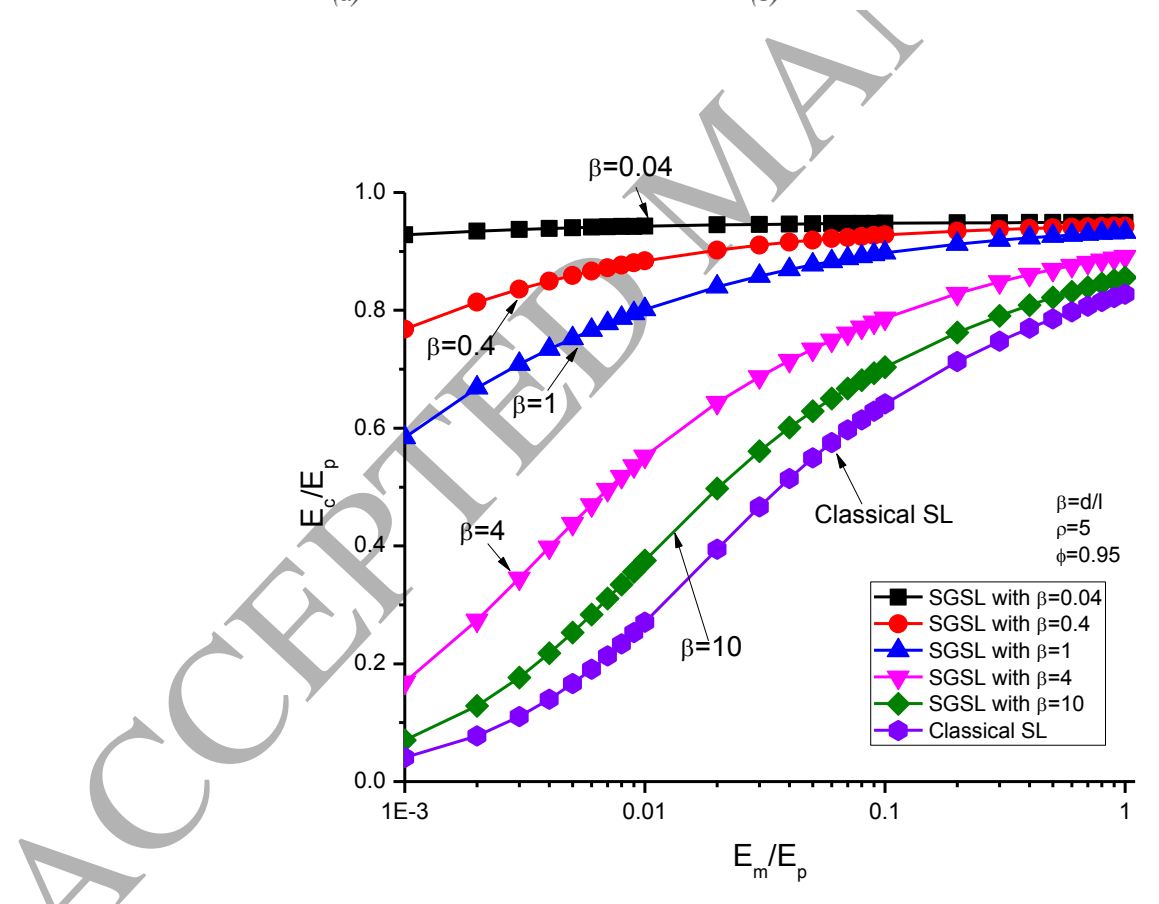

